\title{
Long-Wavelength Excitations in a Bose Gas at Zero Temperature
}

\author{
VICTOR K. WONG* \\ Department of Physics, Unitersity of Michigan, Ann Arbor, Michigan 48104
}

\author{
AND \\ HARVEY GOULD ${ }^{\dagger}$ \\ Department of Physics, Clark University, Worcester, Massachusetts 01610
}

Received January 25, 1973

The long-wavelength excitations in a simple model of a dilute Bose gas at zero temperature are investigated from a purely microscopic viewpoint. The role of the interaction and the effects of the condensate are emphasized in a dielectric formulation, in which the response functions are expressed in terms of regular functions that do not involve an isolated single-interaction line nor an isolated single-particle line. Local number conservation is incorporated into the formulation by the generalized Ward identities, which are used to express the regular functions involving the density in terms of regular functions involving the longitudinal current. A perturbation expansion is then developed for the regular functions, producing to a given order in the perturbation expansion an elementary excitation spectrum without a gap and simultaneously response functions that obey local number conservation and related sum rules.

Explicit results to the first order beyond the Bogoliubov approximation in a simple one-parameter model are obtained for the elementary excitation spectrum $\omega_{k}$, the dynamic structure function $\mathscr{H}(\mathbf{k}, \omega)$, the associated structure functions $\mathscr{S}_{m}(\mathbf{k})$, and the one-particle spectral function $\mathscr{A}(\mathbf{k}, \omega)$, as functions of the wavevector $k$ and frequency $\omega$. These results display the sharing of the gapless spectrum $\omega_{k}$ by the various response functions and are used to confirm that the sum rules of interest are satisfied. It is shown that $\omega_{k}$ and some of the $\mathscr{S}_{m}(\mathbf{k})$ are not analytic functions of $k$ in the long wavelength limit. The dynamic structure function $\mathscr{H}(\mathbf{k}, \omega)$ can be conveniently separated into three parts: a one-phonon term which exhausts the $f$ sum rule, a backflow term, and a background term. The backflow contribution to the static structure function $\mathscr{S}_{0}(\mathbf{k})$ leads to the breakdown of the one-phonon Feynman relation at order $k^{3}$. Both $\mathscr{S}(\mathbf{k}, \omega)$ and $\mathscr{A}(\mathbf{k}, \omega)$ display broad backgrounds because of two-phonon excitations. Simple arguments are given to indicate that some of the qualitative features found for various physical quantities in the first-order model calculation might also be found in superfluid helium.

* Supported in part by a Faculty Research Grant and Fellowship from the Horace H. Rackham School of Graduate Studies at the University of Michigan.

+ Supported in part by the Clark University Research Fund. 


\section{INTRODUCTION}

There are many assumptions that are made in theoretical [1,2] and experimental [3] investigations about the nature of the long wavelength excitations of superfluid helium. In the absence of a tractable microscopic theory of a strongly interacting Bose system, many of these assumptions have not been given a firm theoretical basis for superfluid helium. For this reason we are motivated to perform a rigorous microscopic analysis of the long-wavelength excitations of a simple model of a dilute Bose gas at zero temperature. Although the results of such a study are not directly applicable to superfluid helium, they may be instructive in improving the existing phenomenological theories.

Many previous perturbation calculations of Bose systems have been based on the usual Green's function formulation [4-6], in which the emphasis is on the single-particle propagator. Approximations are usually made on the single-particle self energy and the single-particle spectrum is found from the pole of the singleparticle propagator. The density propagator is separately calculated and the density spectrum is found from the pole of the density propagator. For a given approximation in the single-particle self energy, two unphysical features generally appear. First the single-particle spectrum and the density spectrum do not coincide [6] and second either the conservation laws are violated or the gapless condition on the single-particle spectrum is not satisfied [1,4-6]. Furthermore calculations beyond the well known Bogoliubov approximation have been viewed as being tedious due to the appearance of spurious divergences [4].

In this paper we overcome these difficulties by using a dielectric formulation $[7,8]$ which emphasizes the role of the interaction and the effects of the condensate. By analyzing the structure of the diagrams, we can express the zero-helicity response functions ${ }^{1}$ (i.e., the single-particle, density, and longitudinal-current response functions) in terms of regular functions that do not involve an isolated single-interaction line nor an isolated single-particle line. In particular the denominators of the zero-helicity response functions can be related to the dielectric function. The elementary excitation spectrum is then found from the zero of the dielectric function. In this way the first difficulty mentioned above is eliminated. To remove the second diffculty we build into the formulation the continuity equation via the generalized Ward identities, which are used to express the density functions in terms of the longitudinal-current functions. For example, the dielectric function and the self energy can be expressed in terms of the regular self-energy functions and the regular longitudinal-current functions. A perturbation expansion is then developed for these regular functions, producing zero-helicity response

\footnotetext{
${ }^{1}$ For reasons that will become clear later (Section 2B), we refer to the longitudinal-current, density, and single-particle response functions collectively as zero-helicity response functions.
} 
functions that satisfy the number conservation laws and related sum rules. Furthermore, since the regular longitudinal-current functions are better behaved in the long wavelength limit than their density counterparts, the present approach also enables us to make explicit calculations beyond the Bogoliubov approximation with relative ease and without spurious divergences.

The present first-order calculation beyond the Bogoliubov approximation can be compared with some of the previous calculations in terms of the discussion in Ref. [6]. Since the present single-particle spectrum coincides with the density spectrum, the present first-order calculation cuts across the catcgorization of perturbation calculations found in Ref. [6], which is based on self-energy approximations. In particular the present single-particle spectrum is equivalent to that calculated by Beliaev [4], and the present density spectrum is equivalent to that calculated in the Giradeau-Arnowitt approximation [6]. (See Fig. 5 in Ref. [6].) The difference between previous calculations and the present calculation is that in the usual Green's function formulation the single-particle self energy is approximated, in contrast to the present formulation in which the regular functions that enter into the zero-helicity response functions are approximated. Hence the dielectric formulation is not just a transcription of the usual Green's function formalism, but is a formulation that displays clearly the outstanding feature of a Bose system, viz., all zero-helicity response functions have the same poles, and gives a natural way to obtain approximations that yield both a gapless elementary excitation spectrum and consistent response functions.

In Section 2 we discuss the qualitative features of a Bose system at zero temperature, specify the basic definitions and response functions of interest, review the dielectric formulation of a Bose system, and derive the generalized Ward identities.

We consider a simple model of a Bose gas in Section 3, which can be characterized by a dimensionless parameter $g$. The generalized Ward identities are used to express the regular functions involving the density in terms of regular functions that involve the longitudinal current. These regular functions are formally expanded to first order in $g$. In this way local number conservation is automatically satisfied to first order in $g$.

The first-order calculation is of considerable interest since it represents the simplest approximation in which multiphonon processes appear. We consider in detail the first-order calculation in Section 4 and obtain numerical results for the elementary excitation spectrum $\omega_{k}$, the dynamic structure function $\mathscr{S}(\mathbf{k}, \omega)$, the associated structure functions $\mathscr{S}_{m}(k)=\int_{0}^{\infty} d \omega \omega^{m} \mathscr{S}(\mathbf{k}, \omega)$, and the one-particle spectral function $\mathscr{Z}(\mathbf{k}, \omega)$. The results are used to confirm that the sum rules of interest are satisfied and to explicitly display the sharing of the singularities by the zero-helicity response functions. Both spectral functions $\mathscr{A}(\mathbf{k}, \omega)$ and $\mathscr{T}(\mathbf{k}, \omega)$ display the multiphonon background and threshold. It is found that $\mathscr{F}(\mathbf{k}, \omega)$ 
can be conveniently separated into three terms: a one-phonon term that exhausts the $f$ sum rule, a term due to backflow, and a background term. The backflow contribution to the static structure functions $\mathscr{S}_{0}(k)$ leads to the breakdown of the one-phonon Feynman relation $\mathscr{Y}_{0}(k)=k^{2} / 2 m \omega_{k}$ at order $k^{3}$. It is also seen that $\omega_{k}$ and other functions are not analytic functions of the wavevector $k$ in the long wavelength limit. A brief summary of some of these results has been reported earlier $[9,10]$.

Finally in Section 5 we consider whether some of the qualitative features of various physical quantities found in the first-order model calculations might also be found in superfluid helium. We give general arguments to indicate that $\omega_{k}$ is not an analytic function of $k$ and present simple sum rule arguments to show that the Feynman relation breaks down at order $k^{3}$.

We emphasize that the purpose of the present work is to explicitly display in a rigorous and consistent fashion many qualitative features of a simple but nontrivial model of a Bose system at zero temperature. The numerical coefficients obtained in the model calculation are of little physical interest.

\section{Dielectric Formulation}

It is well known that in general a formalism based upon the dielectric function is convenient for treating the density fluctuations at long wavelengths. Due to the presence of a condensate in a Bose system, the density fluctuations are directly related to the single-particle amplitude fluctuations, and thus the one-particle Green's function is also closely related to the dielectric function. The dielectric formulation $[7,8]$ of a neutral Bose gas has been considered by several workers but has been applied to model calculations [8-11] only recently. To emphasize the physical features and mathematical structure of the formalism, we review in this section the dielectric formulation of a Bose system. In Section 2A we introduce the usual field theoretic formalism of a Bose system. We define in Section 2B the various response functions that will be useful in the following calculation and present a simple physical argument for the relation of the single-particle response function to the dielectric function. In Section $2 \mathrm{C}$ we relate the zero-helicity response functions to the dielectric function and write down the generalized Ward identities that are based on the continuity equation. It will be seen that the dielectric formulation of a Bose system displays in a conspicuous fashion the sharing of the singularities by the zero-helicity response functions and the dominant role of the interaction in long-wavelength propagation.

\section{A. The Hamiltonian}

We consider a system of $N$ spinless Bosons of mass $m$ enclosed in a volume $V$ with the density $n==N / V$ a fixed constant. The Hamiltonian of the system is 


$$
H=\sum_{\mathbf{k}}\left(\epsilon_{k}-\mu\right) a_{\mathbf{k}}^{+} a_{\mathbf{k}}+\frac{1}{2 V} \sum_{\mathbf{k}, \mathbf{k}^{\prime}, \mathbf{q}} v_{q} a_{\mathbf{k}+\mathbf{q}}^{+} a_{\mathbf{k}}{ }^{+} a_{\mathbf{k}^{\prime}+\mathbf{q}} a_{\mathbf{k}},
$$

where $\hbar$ is set equal to unity, $\epsilon_{k}=k^{2} / 2 m, v_{q}=\int d \mathbf{r} e^{i q \cdot r} v(r)$ is the fourier transform of the interparticle potential $v\left(\left|\mathbf{r}_{2}-\mathbf{r}_{1}\right|\right), \mu$ is the chemical potential, and $a_{\mathrm{k}}$ and $a_{\mathrm{k}}{ }^{+}$are the usual boson destruction and creation operators.

The difficulties associated with the macroscopically occupied zero-momentum state, the condensate, can be eliminated by the replacement

$$
a_{0}^{+}=a_{0}=n_{0}^{1 / 2},
$$

where the $c$-number $n_{0}$ is the condensate density and the volume has been set equal to unity. We ignore the possibility that the condensate can have structure. The replacement (2.2) effectively removes the condensate from the dynamics of the system and relegates the condensate to a classical external field. The parameter $n_{0}$ is determined by the condition that the ground state energy shall be a minimum at the correct value of $n_{0}$

$$
\left(\partial / \partial n_{0}\right)\langle H\rangle=0 .
$$

The brackets $\langle\cdots\rangle$ will always denote the ground-state average. The chemical potential $\mu$ is determined by the condition

$$
-(\partial / \partial \mu)\langle H\rangle=n .
$$

The total density of particles not in the condensate is denoted by $n^{\prime}$

$$
n^{\prime}=n-n_{0} .
$$

If we substitute (2.2) into (2.1), we find a new Hamiltonian $H\left(n_{0}\right)$ that depends on the parameter $n_{0}$ and has four distinct types of interaction terms as shown in Fig. 1. Note that the interaction can create and destroy particles with nonzero momentum, i.e., noncondensate particles. With the replacement (2.2), the ground state of the noninteracting Bose system has no particles and is formally a vacuum. We now have a well defincd ficld-theoretic model.

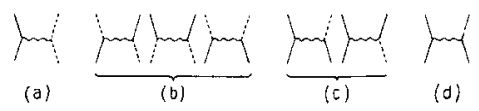

FIG. 1. The four distinct types of interaction terms. (a) The classical field term. (b) The propagation terms. (c) The emission and absorption terms. (d) The noncondensate term. The wavy lines represent the interaction $v$, the solid line represents a noncondensate particle, and the dotted line represents a condensate particle. The interaction term involving no noncondensate particles has been omitted. 


\section{B. The Condensate and the Response Functions}

Many of the interesting physical properties of the system may be summarized in the two-point response function

$$
\mathscr{R}_{X X^{\prime}}(\mathbf{k}, \omega)=-i \int d t e^{i \omega t}\left\langle\left[X_{\mathbf{k}}(t), X_{-\mathbf{k}}^{\prime}\right]\right\rangle \theta(t)
$$

where $X_{\mathbf{k}}, X_{-\mathbf{k}}$ are dynamical variables such as the amplitude, density, and current, and the momentum $\mathbf{k}$ specifies the transformation property of the operators under translation. Writing $\mathscr{R}$ as a sum over matrix elements of $X, X^{\prime}$ between energy eigenstates, we have

$$
\mathscr{R}_{X X^{\prime}}(\mathbf{k}, \omega)=\sum_{n}\left\langle 0\left|X_{\mathbf{k}}\right| n^{\prime}\left\langle n\left|X_{-\mathbf{k}}^{\prime}\right| 0\right\rangle\left(\frac{1}{\omega-E_{n 0}}-\frac{1}{\omega+E_{n 0}}\right),\right.
$$

where $|0\rangle$ is the ground state with zero energy. We see that $\mathscr{R}_{X X^{\prime}}$ has poles at the energies $E_{n 0}$ of the excited states. In all response functions, we write $\omega$ but interpret it as $\omega+i 0+$ whenever it is necessary.

The presence of the condensate in the interacting system gives rise to interaction terms that can emit or absorb particles (Fig. Ic) and thus generate transitions, for example, between one-particle states and two-particle states. It follows that any energy eigenstate is in general a superposition of states of a different number of particles and that the transitions between two states with any number of particles is allowed unless it is forbidden by translational and rotational symmetry. The excited states can then be classified in accordance to the momentum $\mathbf{k}$ and the helicity $^{2}$ (angular momentum along the unit $\mathbf{k}$ vector), viz., the transformation properties under translations and rotations. From (2.7), we see that response functions of $X$ and $X^{\prime}$ with the same translational and rotational transformation properties would have the same poles. In other words, the response functions $\mathscr{R}_{X X^{\prime}}$ as a function of complex $\omega$, with the same momentum $\mathbf{k}$ and hclicity as determincd by the transformation properties of $X$ and $X^{\prime}$, must have the same set of singularities. In general different response functions will have different residues or relative weights at the common singularities. This sharing of singularities is an outstanding feature of a Bose system and is not found in Fermi systems.

The dynamical variables of interest include the amplitude operator $a_{\mathbf{k} \mu}$

$$
a_{\mathbf{k} / 2}= \begin{cases}a_{\mathbf{k}} & \mu=+, \\ a_{-\mathbf{k}}+ & \mu=-\end{cases}
$$

"In keeping with common usage, we refer at times to zero-helicity states as longitudinal states, helicity \pm 1 as transverse, etc. 
the density operator $\rho_{\mathbf{k}}$

$$
\begin{aligned}
\rho_{\mathbf{k}} & =\sum_{\mathbf{p}} a_{\mathbf{p}}{ }^{+} a_{\mathbf{p}+\mathbf{k}} \\
& =n_{0}^{1 / 2} a_{\mathbf{k} \mu} \delta_{\mu}+\sum_{\mathbf{p}}{ }^{\prime} a_{\mathbf{p}}{ }^{+} a_{\mathbf{p}+\mathbf{k}} \quad(\mathbf{k} \neq 0)
\end{aligned}
$$

and the current operator

$$
\mathbf{J}_{\mathbf{k}}=(\mathbf{k} / 2 m) n_{0}^{1 / 2} a_{\mathbf{k} \mu} \beta_{\mu}+m^{-1} \sum_{\mathbf{p}}^{\prime}\left(\mathbf{p}+\frac{1}{2} \mathbf{k}\right) a_{\mathbf{p}}{ }^{+} a_{\mathbf{p}+\mathbf{k}}
$$

where $\delta_{\mu}-1, \beta_{\mu}-\operatorname{sgn}(\mu)$, and from hereon repeated Greek indices are summed. The amplitude response function, or the one-particle Green's function, forms a symmetric $2 \times 2$ matrix

$$
\mathscr{G}_{\mu v}(\mathbf{k}, \omega)=-i \int d t e^{i \omega t}\left\langle\left[a_{\mathbf{k} \mu}(t), a_{\mathbf{k} v}^{+}\right]\right\rangle \theta(t) .
$$

Similarly, the density and current response functions are

$$
\begin{gathered}
\mathscr{F}(\mathbf{k}, \omega)=-i \int d t e^{i \omega t}\left\langle\left[\rho_{\mathbf{k}}(t), \rho_{\mathbf{k}}{ }^{+}\right]\right\rangle \theta(t), \\
\mathscr{F}^{i}(\mathbf{k}, \omega)=-i \int d t e^{i \omega t}\left\langle\left[J_{\mathbf{k}}{ }^{i}(t), \rho_{\mathbf{k}}{ }^{\dagger}\right]\right\rangle \theta(t), \\
\mathscr{F}^{i j}(\mathbf{k}, \omega)=-i \int d t e^{i \omega t}\left\langle\left[J_{\mathbf{k}}{ }^{i}(t), J_{\mathbf{k}}^{j+}\right]\right\rangle \theta(t),
\end{gathered}
$$

where the superscripts $i$ and $j$ label the three components $(i, j=x, y, z)$ of $\mathbf{J}$ with the $z$ axis along $\mathbf{k}$.

The amplitude $a_{\mathbf{k} \mu}$, the density $\rho_{\mathbf{k}}$, and the longitudinal current $J_{\mathbf{k}}{ }^{z}$ all have the same translational and rotational transformation properties, viz, the same momentum $\mathbf{k}$ and zero-helicity. The poles of the zero-helicity response functions constructed from $a_{\mathbf{k} \mu}, \rho_{\mathbf{k}}$ and $J_{\mathbf{k}}{ }^{z}$ give the energies of the excited states with momentum $\mathbf{k}$ and zero helicity. Therefore if one finds a pole in $\mathscr{G}_{\mu \nu}(\mathbf{k}, \omega)$, one will find the same pole (with in general a different residue) in $\mathscr{F}(\mathbf{k}, \omega), \mathscr{F}^{z}(\mathbf{k}, \omega)$, and $\overline{\mathscr{F}}{ }^{z z}(\mathbf{k}, \omega)$. This is easily understood in view of $(2.9)$ and $(2.10)$ where it is seen that $\rho_{\mathbf{k}}, J_{\mathbf{k}} z^{z}$ contain terms linear in $a_{\mathbf{k} u}$.

Other response functions of interest include density and current to amplitude functions

$$
\begin{aligned}
& \mathscr{G}_{\mu}(\mathbf{k}, \omega)=-i \int d t e^{i \omega t}\left\langle\left[\rho_{\mathbf{k}}(t), a_{\mathbf{k} \mu}^{+}\right]\right\rangle \theta(t), \\
& \mathscr{C}_{\mu}^{i}(\mathbf{k}, \omega)=-i \int d t e^{i \omega t}\left\langle\left[J_{\mathbf{k}}^{i}(t), a_{\mathbf{k} \mu}^{+}\right]\right\rangle \theta(t) .
\end{aligned}
$$


It is obvious from the above remarks that $\mathscr{C}_{\mu}$ and $\mathscr{C}_{\mu}{ }^{z}$ will have the same singularities as $\mathscr{G}_{\mu \nu}, \mathscr{F}, \mathscr{F}^{z}, \mathscr{F}^{2 z}$.

In addition to the sharing of singularities, the presence of the condensate also gives rise to amplitude propagation that is dominated by the interaction. If we consider the interaction terms in Fig. 1(b) as a space-time picture with the time axis pointing up and the space axis to the right, we see that the amplitude fluctuation propagates via the particle motion (solid lines) and also via the interaction (wavy lines). For small $k$, the particles involved in the diagram move slowly (the solid lines are almost vertical), but the interaction lines are always instantaneous (wavy lines are horizontal) and effectively transfer the disturbance over a distance $a$, the scattering length. Thus the interaction effectively speeds up the propagator from $k / m$ to the speed $s$. In the $k \rightarrow 0$ limit, the speed $s$ remains finite (for finite $a$ ) and the interaction is solely responsible for the finite speed of propagation. Thus we are naturally led to a description of the one-particle Green's function in terms of the dielectric function, which takes into account the modification of the propagation by the medium.

\section{Exact Relations for the Response Functions}

We call a two-point function irreducible if every one of the diagrams contributing to it is an irreducible diagram, i.e., a diagram that is not made out of two pieces, each containing an end point, connected only by a (wavy) single-interaction line. The general reducible diagram is shown in Fig. 2a. The classical field term, Fig. 1a, is considered irreducible in this classification. We call a function proper if each contribution is represented by a proper diagram, i.e., a diagram that is not made out of two parts connected only by a (solid) single-particle line. The general improper diagram is shown in Fig. 2b. Self-energy diagrams are by definition proper but may be reducible. Our aim is to separate out and sum up both types or singular diagrams, the reducible diagrams and the improper diagrams, and work only with regular diagrams (and functions) that are both proper and irreducible. The irreducible functions of $\mathscr{G}_{\mu \nu}, \mathscr{C}_{\mu}, \mathscr{C}_{\mu}{ }^{i}, \mathscr{F}, \mathscr{F}^{i}, \mathscr{F}^{i j}$ are denoted by capital letters $G_{u v}, C_{u}, C_{u}{ }^{i}, F, F^{i}, F^{i j}$. The regular functions will be further distinguished if necessary by a superscript $r$.

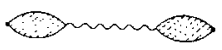

(a)

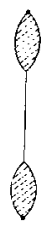

(b)

Frg. 2. Singular diagrams for a two-point response function: (a) reducible diagrams and (b) improper diagrams.

$595 / 83 / 2-2$ 
We first consider the response functions containing the density and/or current. The density response function $\mathscr{F}$ can be expressed in terms of its irreducible function $F$ by summing the geometric series in Fig. 3

$$
\mathscr{F}=\frac{F}{1-v F}=\frac{F}{\epsilon},
$$

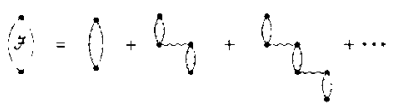

FIG. 3. The density (or current) response function $\mathscr{F}$ in terms of irreducible functions. A heavy dot represents a density (or current) vertex and the shaded diagrams are irreducible.

where we have defined the dielectric function $\epsilon(\mathbf{k}, \omega)$ by the relation $\epsilon=1-v F$. The cylindrical symmetry about $\mathbf{k}$ leads to the conclusions that

$$
\mathscr{C}_{\mu}{ }^{x}=\mathscr{C}_{\mu}{ }^{y}=\mathscr{F}^{x}=\mathscr{F}^{y}=\mathscr{F}^{x y}=\mathscr{F}^{x z}=\mathscr{F}^{y z}=0,
$$

and furthermore that the transverse-current response functions are all regular. An analysis similar to (2.17) shows that the longitudinal-current response functions satisfy

$$
\begin{aligned}
\mathscr{F}^{z} & =F^{z} / \epsilon \\
\mathscr{F}^{z z} & =F^{z z}+F^{z} F^{z} v / \epsilon,
\end{aligned}
$$

and the amplitude to density and current response function satisfy

$$
\begin{aligned}
\mathscr{C}_{\mu} & =C_{\mu} / \epsilon \\
\mathscr{C}_{\mu}{ }^{z} & =C_{\mu}{ }^{2}+F^{z} C_{\mu} v / \epsilon
\end{aligned}
$$

The proper part of the $F$ 's can be separated as in Fig. 4, and the corresponding equations are

$$
\begin{gathered}
F=\Lambda_{\mu} G_{\mu \nu} \Lambda_{\nu}+F^{r}, \\
F^{z}=\Lambda_{\mu}{ }^{z} G_{\mu \nu} \Lambda_{v}+F^{z r}, \\
F^{z z}=\Lambda_{\mu}{ }^{z} G_{\mu \nu} \Lambda_{v}{ }^{z}+F^{z z r}, \\
F
\end{gathered}
$$

Fig. 4. The irreducible density (or current) response function $F$ in terms of regular functions. A heavy dot represents density (or current) vertex and the cross-hatched diagrams are regular. 
where the vertex functions $A$ are defined as

$$
\begin{aligned}
C_{\mu} & =\Lambda_{\nu} G_{\nu \mu}, \\
C_{\mu}{ }^{z} & =\Lambda_{\nu}{ }^{z} G_{\nu \mu} .
\end{aligned}
$$

Here $F^{r}, F^{z r}, F^{z z r}, \Lambda_{\mu}, \Lambda_{\mu}^{z}$, are all regular functions.

The response functions containing the density $\rho_{\mathbf{k}}$ and/or the longitudinal current $J_{\mathbf{k}}{ }^{z}$ can be related to each other via the continuity equation

$$
i \partial \rho_{\mathbf{k}} / \partial t=k J_{\mathbf{k}}{ }^{2} .
$$

By taking time derivatives of the response functions, we can derive using (2.28) the relations

$$
\begin{aligned}
\omega \mathscr{F} & =k \mathscr{F}^{z}, \\
\omega \mathscr{F}^{z} & =k\left(\mathscr{F}^{z z}+n / m\right), \\
\omega^{2} \mathscr{F} & =k^{2}\left(\mathscr{F}^{z z}+n / m\right),
\end{aligned}
$$

which remain valid when the $\mathscr{F}$ 's are replaced by the irreducible $F$ 's. We have for the $\mathscr{C}$ 's

$$
\omega \mathscr{C}_{\mu}=k \mathscr{C}_{\mu}{ }^{z}+n_{0}^{1 / 2} \beta_{\mu},
$$

where $\beta_{\mu}=\operatorname{sgn}(\mu)$. Again (2.32) is valid with $\mathscr{C} \rightarrow C$, and using (2.26) and (2.27), we can write

$$
\omega \Lambda_{\nu}=k \Lambda_{\nu}{ }^{2}+n_{0}^{1 / 2} \beta_{\mu} G_{\mu \nu}^{-1} .
$$

Finally from the definition of $F^{r}, F^{z r}, F^{z z r}$, and (2.29) and (2.33) we have

$$
\begin{aligned}
\omega F^{r} & =k F^{z r}-n_{0}^{1 / 2} \Lambda_{\mu} \beta_{\mu}, \\
\omega F^{r} & =k\left(F^{z z r}+n / m\right)-n_{0}^{1 / 2} \Lambda_{\mu}{ }^{z} \beta_{\mu}, \\
\omega^{2} F^{r} & =k^{2}\left(F^{z z r}+n / m\right)-n_{0}^{1 / 2}\left(k \Lambda_{\mu}{ }^{z}+\omega \Lambda_{\mu}\right) \beta_{\mu} .
\end{aligned}
$$

These identities derived from the continuity equation are analogous to the Ward identities in field theory and will be referred to as the "generalized Ward identities" $[1,6,12]$.

We now analyze the one-particle Green's function. The Green's function $\mathscr{G}_{\mu \nu}$ can be expressed in terms of the (proper) self energy $\mathscr{M}_{\mu \nu}$ by the relation

$$
\mathscr{G}_{\mu \nu}(\mathbf{k}, \omega)=\frac{1}{\mathscr{D}(\mathbf{k}, \omega)} \mathscr{N}_{\mu \nu}(\mathbf{k}, \omega),
$$


where

and

$$
\begin{aligned}
& \mathscr{N}_{++}=\omega+\epsilon_{k}-\mu+\mathscr{H}_{--}, \\
& \mathscr{N}_{+-}=-\mathscr{M}_{+-}=-\mathscr{M}_{\mathbf{2}}, \\
& \mathscr{N}_{--}=-\omega+\epsilon_{k}-\mu+\mathscr{M}_{++},
\end{aligned}
$$

$$
\begin{aligned}
& \mathscr{D}=(\omega-\mathscr{A})^{2}-\left(\epsilon_{k}-\mu+\mathscr{S}-\mathscr{M}_{2}\right)\left(\epsilon_{k}-\mu+\mathscr{S}+\mathscr{M}_{2}\right), \\
& \mathscr{S}=\frac{1}{2}\left(\mathscr{M}_{++}+\mathscr{M}_{--}\right) ; \quad \mathscr{N}=\frac{1}{2}\left(\mathscr{M}_{++}-\mathscr{M}_{--}\right) .
\end{aligned}
$$

If we define $G_{\mu \nu}, N_{\mu \nu}, D, M_{\mu \nu}, S, A, M_{2}$ as the corresponding irreducible functions, the equations (2.37)-(2.39) remain valid with script letters replaced by capital letters. These irreducible functions are all regular functions since $M_{\mu v}$ is proper. The chemical potential is determined from the Hugenholtz-Pines [5] theorem, which can be expressed in terms of the irreducible self energy at $k=\omega=0$

$$
\mu=S(0,0)-M_{2}(0,0) .
$$

To sce this, we cxpress $\mathscr{M}_{\mu \nu}$ in terms of its irreducible $M_{\mu \nu}$ via Fig. 5

$$
\begin{aligned}
& \mathscr{H}_{u v}=M_{\mu v}+\Lambda_{\mu} \Lambda_{v} v / \epsilon^{r}, \\
& \epsilon^{r}=1-v F^{r} \text {. }
\end{aligned}
$$

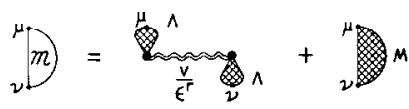

FIG. 5. The (proper) self-energy in terms of regular functions. The double wavy line represents the proper effective interaction $v / \epsilon^{r}$ and the cross-hatched diagrams are regular.

Since $\mathscr{M}$ is proper by definition, the dielectric function that enters into (2.41) must be expressed in terms of the proper $F^{r}$. From (2.38), (2.39), and (2.41) we find

$$
\mathscr{S}-\mathscr{M}_{2}=S-M_{2}+\frac{1}{2}\left(v / \epsilon^{r}\right)\left(\Lambda_{\mu} \beta_{\mu}\right)^{2} .
$$

Using the fact [1] that in the $(k \rightarrow 0, \omega \rightarrow 0)$ limit $F^{r} \rightarrow-\partial n^{\prime} / \partial \mu$ and $F^{z r} \rightarrow 0$, we see from (2.34) and (2.42) that the Hugenholtz-Pines theorem, usually expressed as $\mu=\mathscr{S}(0,0)-\mathscr{M}_{2}(0,0)$, can be written as in (2.40). Substituting (2.41) into (2.38), we find

$$
\mathscr{A}_{\mu \nu}=N_{\mu \nu}+\operatorname{sgn}(\mu) \operatorname{sgn}(\nu) \Lambda_{-\mu} \Lambda_{-\nu} v / \epsilon^{r},
$$

where both $\mu$ and $\nu$ are indices and no summation over $\mu$ and $\nu$ is implied. Substituting (2.41) into (2.39), we find after some algebra

$$
\mathscr{D}=D \epsilon / \epsilon^{r} \text {. }
$$


From (2.37), (2.43), and (2.44), we see that

$$
\mathscr{G}_{\mu v}=\frac{1}{\epsilon}\left[\epsilon^{r} G_{\mu v}+\operatorname{sgn}(\mu) \operatorname{sgn}(\nu) \Lambda_{-\mu} \Lambda_{-v} v / D\right] .
$$

Thus the dielectric function plays an essential role in the structure of the oneparticle Green's function in a Bose system. In the above we have analyzed the role of the isolated single-interaction line and that of the isolated single-particle line by summing a few geometric series, and in this way have been able to express the response functions and the dielectric function in terms of the regular functions $\Lambda_{\mu}, \Lambda_{\mu}^{z}, M_{\mu \nu}, F^{r}, F^{z r}$, and $F^{z z r}$. The analysis of the isolated pair of single-particle lines leads to equations of the Bethe-Salpeter type and has been considered by $\mathrm{Ma}[11]$.

\section{Perturbation Expansion}

We choose a simple model of a Bose gas and assume that the short-range two body interaction $v(k)$ can be summarized by the $s$-wave scattering length $a$ so that $v(k)$ is a constant. In general the scattering length alone is not sufficient to determine the interaction potential, and our simple model will not illuminate effects pertaining to the detailed form of the interaction. To the order of approximation of interest the parameter $v$ can be eliminated by using the perturbation expansion for the scattering length $a$ in powers of $v$. We thus regard the quantities $m, n$, and $a$ as the input parameters of the theory.

We now develop a perturbation expansion for the zero-helicity response functions. The consideration of the nature of the zeroth (Bogoliubov) approximation in Section $3 \mathrm{~A}$ enables us to discuss the natural units and the relative order of magnitude of the various diagrammatic contributions (Section 3B). In Section 3C we utilize the generalized Ward identities (2.29)-(2.31) to express the regular functions involving the density in terms of the regular functions involving the longitudinal current. By expanding these regular functions, we build in to each order the local number conservation law. The advantages of this procedure will be made explicit in Section 4.

\section{A. Zeroth Approximation}

The zeroth approximation in our simple Bose model corresponds to the well known Bogoliubov approximation. Instead of focusing on the self energy $\mathscr{A}$ of the one-particle Green's function as in the usual treatments [4-6], we center our attention on the regular functions, e.g., $\Lambda_{\mu}^{z}, F^{z z r}, M_{\mu v}$, etc. Our approach is to consider the simplest approximation that is consistent with the sharing of singularities and that incorporates the dominant role of the interaction on longwavelength propagation. 
First, we specify the zeroth approximation as one in which $a_{\mu \mathbf{k}}, \rho_{\mathbf{k}}$, and $J_{\mathbf{k}}$ are identical up to some proportionality constant, or equivalently, all the two-amplitude terms in $\rho_{\mathbf{k}}$ and $J_{\mathbf{k}}(2.9)$ and $(2.10)$ are ignored

$$
\begin{aligned}
& \rho_{\mathbf{k}}=n_{\mathbf{0}}^{1 / 2} a_{\mathbf{k} \mu} \delta_{\mu} \quad(k \neq 0), \\
& J_{\mathbf{k}}=n_{0}^{1 / 2}(k / 2 m) a_{\mathbf{k} \mu} \beta_{\mu} .
\end{aligned}
$$

Comparing (3.1) and (3.2) with (2.9) and (2.10), we note that to this order there is no depletion, $n^{\prime}=n \quad n_{0}=0$, and there is no transverse response, $J_{\mathbf{k}}=J_{\mathbf{k}}{ }^{z}$. From (3.1) and (3.2) it follows that the vertex functions are given by

$$
\begin{aligned}
\Lambda_{\mu} & =n_{0}^{1 / 2} \delta_{\mu}, \\
\Lambda_{\mu}{ }^{z} & =n_{0}^{1 / 2}(k / 2 m) \beta_{\mu},
\end{aligned}
$$

and the regular $F$ 's all vanish

$$
F^{r}=F^{z r}=F^{z z r}=0 .
$$

Since the irreducible self energy $M_{\mu \nu}$ is related to the amplitude response function, it must be specified independently of (3.1) and (3.2). It is apparent from (2.37) and (2.40) that $M_{\mu \nu}$ only appears in the form $M_{\mu \nu}-\mu \delta_{\mu \nu}$, where $\mu$ (not to be confused with the indices) is the chemical potential and $\delta_{\mu \nu}$ is the Kronecker delta function. The lowest order diagram for $M_{\mu \nu}$ is the external classical-field diagram (Fig. 1a), which gives just an energy shift, $M_{++}=M_{--}=n v, M_{+-}=M_{-+}=0$. But from (2.40) in lowest order, there is a corresponding shift in the chemical potential, $\mu=n v$. Hence we have

$$
M_{ \pm \pm}-\mu=M_{ \pm \mp}=0 .
$$

Equations (3.3)-(3.5) completes the specification of the regular functions in the zeroth approximation. Note that nowhere in (3.3)-(3.5) does the potential appear.

Wc now list the results for the irreducible response functions that follow from (3.3)-(3.5). From (2.37)-(2.39), we see that the irreducible Green's function $G_{\mu \nu}$ is diagonal in the zeroth order

$$
\begin{aligned}
& G_{++}^{(0)}=\left(\omega-\epsilon_{k}\right)^{-1}, \\
& G_{-\ldots}^{(0)}=-\left(\omega+\epsilon_{k}\right)^{-1} .
\end{aligned}
$$

From (2.23)-(2.25), we can write the irreducible $F^{(\mathbf{0})}$ as

$$
\begin{aligned}
F^{(0)} & =\left(n k^{2} / m\right)\left(\omega^{2}-\epsilon_{k}\right)^{-1}, \\
F^{z(0)} & =(n k \omega / m)\left(\omega^{2}-\epsilon_{k}^{2}\right)^{-1}, \\
F^{z z(0)} & =\left(n \epsilon_{k}^{2} / m\right)\left(\omega^{2}-\epsilon_{k}^{2}\right)^{-1} .
\end{aligned}
$$


From (2.26) and (2.27), we find

$$
\begin{aligned}
& C_{\mu}^{(0)}=n^{1 / 2}\left(\omega \beta_{\mu}+\epsilon_{k} \delta_{\mu}\right)\left(\omega^{2}-\epsilon_{k}^{2}\right)^{-1}, \\
& C_{\mu}^{z(0)}=n^{1 / 2}(k / 2 m)\left(\omega \beta_{\mu}+\epsilon_{k} \delta_{\mu}\right)\left(\omega^{2}-\epsilon_{k}^{2}\right)^{-1} .
\end{aligned}
$$

In zeroth order, the irreducible response functions all have poles at the freeparticle spectrum $\epsilon_{k}$ and are in fact the response functions for the ideal Bose gas.

In order to incorporate the dominant role of the interaction on long-wavelength propagation, we retain the lowest order term in $\epsilon(\mathbf{k}, \omega)-1=v F(\mathbf{k}, \omega)$. Using (3.7), we obtain the dielectric function

$$
\epsilon^{(0)}(\mathbf{k}, \omega)=\left(\omega^{2}-\omega_{k}^{2(0)}\right)\left(\omega^{2}-\epsilon_{k}^{2}\right)^{-1},
$$

where the Bogoliubov spectrum $\omega^{(0)}$ is given by

$$
\begin{gathered}
\omega_{k}^{2(0)}=s_{0}{ }^{2} k^{2}+\epsilon_{k}{ }^{2}, \\
s_{0}{ }^{2}=n v / m=4 \pi n a / m^{2} .
\end{gathered}
$$

We now list the results for the response functions that can be obtained with the aid of (3.9). From $(2.17),(2.19)$, and (2.20), we find

$$
\begin{aligned}
\mathscr{F}^{(0)} & =\left(n k^{2} / m\right)\left(\omega^{2}-\omega_{k}^{2(0)}\right)^{-1}, \\
\mathscr{F}^{z(0)} & =(n k \omega / m)\left(\omega^{2}-\omega_{k}^{2(0)}\right)^{-1}, \\
\mathscr{F}^{z z(0)} & =\left(n \omega_{k}^{2(0)} / m\right)\left(\omega^{2}-\omega_{k}^{2(0)}\right)^{-1} ;
\end{aligned}
$$

and from (2.21) and (2.22) we have

$$
\begin{aligned}
& \mathscr{C}_{\mu}^{(0)}=n^{1 / 2}\left(\omega \beta_{\mu}+\epsilon_{k} \delta_{\mu}\right)\left(\omega^{2}-\omega_{2}^{2(0)}\right)^{-1} \\
& \mathscr{C}_{\mu}^{z(0)}=n^{1 / 2}\left(\omega_{k}^{2(0)} \beta_{\mu}+\omega \epsilon_{k} \delta_{\mu}\right)\left(\omega^{2}-\omega_{k}^{2(0)}\right)^{1} .
\end{aligned}
$$

Finally the one-particle Green's function is obtained from (2.45)

$$
\begin{aligned}
\mathscr{G}_{++}^{(0)} & =\frac{u_{k}^{2}}{\omega-\omega_{k}^{(0)}}-\frac{v_{k}{ }^{2}}{\omega+\omega_{k}^{(0)}}, \\
\mathscr{G}_{+-}^{(0)} & =-u_{k} n_{k}\left(\frac{1}{\omega-\omega_{k}^{(0)}}-\frac{1}{\omega+\omega_{k}^{(0)}}\right)
\end{aligned}
$$


where

$$
\begin{aligned}
v_{k}^{2} & =\frac{1}{2}\left[\frac{\epsilon_{k}+m s_{0}{ }^{2}}{\omega_{k}^{(0)}}-1\right], \\
u_{k}{ }^{2} & =\frac{1}{2}\left[\frac{\epsilon_{k}+m s_{0}{ }^{2}}{\omega_{k}^{(0)}}+1\right] \\
u_{k} v_{k} & =\frac{m s_{0}{ }^{2}}{2 \omega_{k}^{(0)}} .
\end{aligned}
$$

Note that the poles of the response functions (3.11)-(3.13) are all located at $\omega^{2}=\omega_{k}^{2(0)}$ and have been shifted to this position by the dielectric function (3.8) from the $\omega^{2}=\epsilon_{k}^{2}$ poles of the irreducible response functions.

\section{B. Units and Magnitudes}

It is convenient to introduce the natural units of momentum and energy in the zeroth order, which are respectively

$$
\begin{aligned}
& k_{0}=m s_{0}=(4 \pi n a)^{1 / 2}, \\
& T_{0}=m s_{0}{ }^{2}=k_{0}{ }^{2} / m=4 \pi n a / m .
\end{aligned}
$$

The small dimensionless parameter $g$ is taken to be

$$
g=4 \pi a k_{0}=(4 \pi a)^{3 / 2} n^{1 / 2} .
$$

If we measure the momentum and energy in terms of $k_{0}$ and $T_{0}$ respectively, we have a new set of natural units in which

$$
\begin{aligned}
k_{0} & =T_{0}=m=s_{0}=1, \\
g & =n^{-1}=4 \pi a .
\end{aligned}
$$

We shall work in these natural units whenever it will not cause confusion.

To obtain the leading order of magnitude of any diagram, we note that (a) each (wavy) interaction line contributes a factor $T_{0} / n_{0} \sim g$; (b) each (solid) one-particle line contributes a factor $T_{0}^{-1} \sim 1$; (c) each (dotted) condensate line contributes a factor of $n_{0}^{1 / 2} \sim g^{-1 / 2}$; (d) each loop of integration contributes a factor of $k_{0}{ }^{3} T_{0} \sim 1$. It is evident that the leading order of magnitude of any diagram is determined by the number of (wavy) interaction lines minus half of the number of (dotted) condensate lines. As an example, consider a self-energy diagram with $n_{v}$ (wavy) interaction lines, $n_{g}$ (solid) one-particle lines, $n_{c}$ (dotted) condensate lines, and $l$ loops of integration. The topology of such a diagram tells us that

$$
\begin{aligned}
\frac{1}{2} n_{c}+l & =n_{v} \\
\frac{1}{2} n_{c}+n_{g}+1 & =2 n_{v} .
\end{aligned}
$$


Thus the leading order of magnitude of any self-energy diagram is given by $g^{i}$. The lowest (zeroth) order is evidently given by the class of diagrams with no loops of integration. The next (first) order is then all diagrams with one loop of integration, or one-loop diagrams, which will be discussed in detail in Section 4.

For clarity we list the order of magnitude of the relevant quantities in zeroth approximation, where only the lowest-order terms in $g$ are kept:

$$
\begin{gathered}
n_{0}=g^{-1}, \quad n^{\prime}=0, \\
v / g=1, \quad M_{++}-\mu=0, \quad M_{+-}=0, \\
G_{u v}=G_{\mu \nu}^{(0)}, \quad N_{\mu \nu}=N_{\mu \nu}^{(0)}, \quad D=D^{(0)}, \\
g^{1 / 2} \Lambda_{u}=\delta_{\mu}, \quad g^{1 / 2} \Lambda_{i \nu}^{z}=\frac{1}{2} k \beta_{u}, \\
g F=F^{(0)}, \quad g F^{z z}=F^{z z(0)}, \quad F^{r}=F^{z z r}=0, \\
\epsilon=1-F^{(0)}, \quad \epsilon^{r}=1, \\
\mathscr{G}_{\mu \nu}=\mathscr{G}_{\mu \nu}^{(0)}, \quad g \mathscr{\mathcal { H }}=\mathscr{F}^{(0)}, \quad g \mathscr{\mathscr { F }}^{z z}=\mathscr{F}^{z z(0)},
\end{gathered}
$$

We have factored out the $g$ dependence explicitly so that $F^{(0)}, F^{z z(0)}$, etc., are all of order unity.

\section{Higher Order Approximations Using Generalized Ward Identities}

One difficulty associated with previous perturbation expansions is that it was not possible, up to a given order, to obtain an elementary excitation spectrum without a gap and to verify simultaneously certain sum rules based upon the conservation laws. In order to overcome this problem, we include the continuity equation expressing local number conservation into the structure of our formalism by utilizing the generalized Ward identities (2.29)-(2.31). The Green's function and the density response function can then be reexpressed in terms of the regular functions involving the longitudinal current (i.e., $A_{\mu}{ }^{z}$ and $F^{z z r}$ ) and $M_{\mu \nu}$. By approximating these regular functions, certain sum rules for the density and current response functions are automatically satisfied to that order of approximation. In this section we derive the formal expressions for the response functions to the first approximation beyond the zeroth (Bogoliubov) approximation. We show explicitly in Section 4 that the first approximation gives an elementary excitation spectrum without a gap and satisfies the well known sum rules.

We first express the density response function in terms of $M_{\mu \nu}, A_{\mu}^{z}$, and $F^{z z r}$, and then expand these regular functions to first order in $g$. Our starting equation (2.17) can be written as

$$
g \mathscr{F}(\mathbf{k}, \omega)=\left[(g F(\mathbf{k}, \omega))^{-1}-v j g\right]^{-1},
$$


which can be cast with the aid of the identity (2.31) into the form

$$
g \mathscr{F}(\mathbf{k}, \omega)=k^{2}\left[\omega^{2}\left(1+g F^{z z}(\mathbf{k}, \omega)\right)^{-1}-k^{2} v / g\right]^{-1}
$$

The function $F^{z z}$ can be expressed in terms of regular functions by using (2.28)

$$
g F^{z z}=g \Lambda_{\mu}^{z} G_{\mu \nu} \Lambda_{\nu}^{z}+g F^{z z r}
$$

The remaining task is to systcmatically expand both sides of (3.20) and (3.21) to first order in $g$; for example,

$$
g^{\mathscr{F}}(\mathbf{k}, \omega)=\mathscr{F}^{(0)}(\mathbf{k}, \omega)+g^{\mathscr{F}}{ }^{(1)}(\mathbf{k}, \omega)+O\left(g^{2}\right) .
$$

Although the coupling constant $g$ is taken to be small, the expansion (3.22) should be applied with caution in the limit of small or large $\omega$. The point is that, in general, the limits $g \rightarrow 0$ and $\omega \rightarrow 0$ or $\omega \rightarrow \infty$ are not interchangeable. Keeping this in mind, we suppress the $\mathbf{k}$ and $\omega$ dependences in the following expansions through $O(g)$

$$
\begin{aligned}
g^{1 / 2} A_{\mu}^{z} & =\frac{1}{2} k \beta_{\mu}\left(1-\frac{1}{2} g h^{\prime(1)}\right)+g A_{\mu}^{z(1)}+O\left(g^{2}\right) \\
g n^{\prime} & =g n^{\prime(1)}+O\left(g^{2}\right) \\
G_{\mu \nu} & =G_{\mu \nu}^{(0)}+g G_{\mu \nu}^{(1)}+O\left(g^{2}\right) \\
g F^{z z r} & =g F^{z z r(1)}+O\left(g^{2}\right) \\
v / g & =1+g v^{(1)}+O\left(g^{2}\right) .
\end{aligned}
$$

The irreducible Green's function $G_{\mu \nu}$ can be expressed in terms of the various irreducible self-energy functions by using the relations (2.37)-(2.39). We write

$$
\begin{gathered}
G^{(0)}=N_{\mu \nu}^{(0)} / D^{(0)}, \\
N_{ \pm \pm}^{(0)}= \pm \omega+\epsilon_{k} ; \quad N_{+-}^{(0)}=0 ; \quad D^{(0)}=\omega^{2}-\epsilon_{k}{ }^{2},
\end{gathered}
$$

and

$$
\begin{gathered}
G^{(1)}=\left[N_{\mu \nu}^{(1)}-D^{(1)} N_{\mu \nu}^{(0)} / D^{(0)}\right] / D^{(0)}, \\
N_{ \pm \pm}^{(1)}=-\mu^{(1)}+M_{\mp \mp}^{(1)} ; \quad N_{+-}^{(1)}=-M_{2}^{(1)}, \\
D^{(1)}=-2 \omega A^{(1)}-k^{2}\left(S^{(1)}-\mu^{(1)}\right) .
\end{gathered}
$$

If we identify the $O(1)$ terms on both sides of (3.20) and (3.21) and use (3.27) and (3.28), we find $\mathscr{F}^{(0)}(\mathbf{k}, \omega)=k^{2}\left(\omega^{2}-\omega_{k}^{2(0)}\right)^{-1}$ in agreement with (3.11). If we collect 
the $O(g)$ terms in the above equations (3.20)-(3.30), we find that after some algebra

$$
\begin{aligned}
\mathscr{F}^{(1)}(\mathbf{k}, \omega)= & \left(k^{2} / \omega^{2}\right)\left(\omega^{2}-\omega_{k}^{2(0)}\right)^{-2} \mathscr{N}^{z z(1)}(\mathbf{k}, \omega), \\
\mathscr{N}^{z z(1)}(\mathbf{k}, \omega)= & \left(\omega^{2}-\epsilon_{k}^{2}\right)^{2} F^{z z(1)}(k, \omega)+\omega^{2} k^{2} v^{(1)}, \\
\mathscr{N}^{z \bar{z}(1)}(\mathbf{k}, \omega)= & \epsilon_{k}\left(\omega^{2}-\epsilon_{k}^{2}\right)\left[S^{(1)}+M_{2}^{(1)}-\mu^{(1)}+2 \omega \delta_{\mu} A_{\mu}^{z(1)} / k\right. \\
& \left.+k \beta_{\mu} A_{\mu}^{z(1)}-\epsilon_{k} n^{(1)}\right] \\
& +k^{2}\left[\omega \epsilon_{k} A^{(1)}+\epsilon_{k}^{2}\left(S^{(1)}-\mu^{(1)}\right)+\omega^{2} \iota^{(1)}\right] \\
& +\left(\omega^{2}-\epsilon_{k}^{2}\right)^{2} F^{z z r(1)} .
\end{aligned}
$$

Equations (3.31) and (3.32) complete the expression of the density response function $\mathscr{F}$ to $O(\mathrm{~g})$.

Alternatively we can expand (3.19) as it stands and obtain

$$
\mathscr{F}^{(1)}(\mathbf{k}, \omega)=\left(\omega^{2}-\omega_{k}^{2(0)}\right)^{-2} \mathscr{N}^{(1)}(\mathbf{k}, \omega)
$$

where

$$
\begin{aligned}
\mathscr{N}^{(\mathbf{1})}(\mathbf{k}, \omega)= & 2\left(\omega^{2}-\epsilon_{k}^{2}\right)\left[S^{(1)}-M_{2}^{(1)}-\mu^{(1)}+\epsilon_{k} \delta_{\mu} \Lambda_{\mu}^{(\mathbf{1})}+\omega \beta_{\mu} \Lambda_{\mu}^{(1)}-\epsilon_{k} h^{(1)}\right] \\
& +2 k^{2}\left[\omega A^{(1)}+\epsilon_{k}\left(S^{(1)}-\mu^{(1)}\right)+\epsilon_{k} v^{(1)}\right]+\left(\omega^{2}-\epsilon_{k}{ }^{2}\right)^{2} F^{r(1)} .
\end{aligned}
$$

By expanding the identities (2.33) and (2.36) to first order in $g$, we can eliminate $\Lambda_{\mu}^{(1)}$ and $F^{r(1)}$ in favor of $\Lambda_{\mu}^{z(1)}$ and $F^{z a r(1)}$ and verify that (3.33) and (3.34) are identical to (3.31) and (3.32).

Next we consider the one-particle Green's function $\mathscr{G}_{++}(\mathbf{k}, \omega)$, which is given by $(2.45)$

$$
\mathscr{G}_{++}(\mathbf{k}, \omega)=\left[\epsilon^{r} G_{++}+\Lambda_{-} \Lambda_{-} v / D\right] / \epsilon .
$$

To make the algebra tractable we consider the two terms on the right side of (3.35) separately. We use the irreducible version of the identity (2.31) to rewrite the dielectric function $\epsilon(\mathbf{k}, \omega)$ as

$$
\epsilon(\mathbf{k}, \omega)=1-\frac{v}{g} \cdot \frac{k^{2}}{\omega^{2}}\left(1+g F^{z z}(\mathbf{k}, \omega)\right)
$$

and use the identities (2.33) and (2.36) to rewrite $\epsilon^{r}$ as

$$
\epsilon^{r}(\mathbf{k}, \omega)=1-\frac{v}{g} \cdot \frac{1}{\omega^{2}}\left[k^{2} g F^{z z r}+k^{2}-2 k n_{0}^{1 / 2} g \Lambda_{\mu}{ }^{z} \beta_{\mu}-n_{0} g \beta_{\mu} G_{\mu v}^{-1} \beta_{v}\right] .
$$


Now we expand (3.36) and (3.37) to first order in $g$, using (3.23) and (3.26) and (3.27) and (3.29). Collecting terms to $O(g)$, we find for the first term of (3.35)

$$
\begin{aligned}
\left(\frac{1}{\epsilon} \epsilon^{r} G_{++}\right)^{(1)}= & \left(k^{2} / \omega^{2}\right)\left(\omega+\epsilon_{k}\right)\left(\omega^{2}-\omega_{k}^{2(0)}\right)^{-2}\left[\omega^{2} l^{(1)}+\left(\omega^{2}-\epsilon_{k}^{2}\right) F^{z z(1)}\right] \\
& +\left(\omega^{2}-\omega_{k}^{2(0)}\right)^{-1}\left[N_{++}^{(1)}-\frac{\omega+\epsilon_{k}}{\omega^{2}-\epsilon_{k}^{2}} D^{(1)}-\frac{\omega+\epsilon_{k}}{\omega^{2}}\right. \\
& \left.\times\left(k^{2} F^{z z r(1)}-2 k \Lambda_{\mu}^{z(1)} \beta_{\mu}+2\left(S^{(1)}-M_{2}^{(1)}-\mu^{(1)}\right)+k^{2} n^{\prime(1)}\right)\right]
\end{aligned}
$$

To expand the second term of (3.35), we first use the identity (2.33) to write

$$
\Lambda_{-} \Lambda_{-}=\omega^{-2}\left[k^{2} \Lambda_{-}^{z} \Lambda_{-}^{z}+2 k \Lambda_{-}^{z} n_{0}^{1 / 2}\left(N_{++}+N_{+-}\right)+n_{0}\left(N_{++}+N_{+-}\right)^{2}\right]
$$

Expanding (3.36), (3.39), and $D$ to first order in $g$, we find the expansion of the second term of $(3.35)$ to $O(g)$

$$
\begin{aligned}
\left(\frac{1}{\epsilon} \Lambda_{-} \Lambda_{-} \frac{v}{D}\right)^{(1)}= & \left(\omega^{2}-\omega_{k}^{2(0)}\right)^{-2}\left[k^{2} v^{(1)}+\left(k^{2} / \omega^{2}\right)\left(\omega^{2}-\epsilon_{k}^{2}\right) F^{z z(1)}\right] \\
& +\left(\omega^{2}-\omega_{k}^{2(0)}\right)^{-1}\left[v^{(1)}-n^{(1)}+2 k \omega^{-1} \Lambda_{-}^{z(1)}\right. \\
& \left.+2 \omega^{-1}\left(N_{++}^{(1)}+N_{+-}^{(1)}\right)-\left(\omega^{2}-\epsilon_{k}^{2}\right)^{-1} D^{(1)}\right]
\end{aligned}
$$

If we combine (3.38) and (3.40), we can write $\mathscr{G}_{++}^{(1)}$ in the form

$$
k^{2} \mathscr{G}_{++}^{(1)}(\mathbf{k}, \omega)=\left(\omega+1+\epsilon_{k}\right) \mathscr{F}^{(1)}(\mathbf{k}, \omega)+\mathscr{H}^{(1)}(\mathbf{k}, \omega)
$$

with

$$
\begin{aligned}
\mathscr{H}^{(1)}(\mathbf{k}, \omega) & =\left(\omega^{2}-\omega_{\hbar}^{2(0)}\right)^{-1} \mathscr{I}^{z z(1)}(\mathbf{k}, \omega), \\
\mathscr{I}^{z z(1)}(\mathbf{k}, \omega) & =\mathscr{I}_{+}^{z z(1)}(\mathbf{k}, \omega)+\mathscr{I}_{-}^{z z(1)}(\mathbf{k}, \omega), \\
\mathscr{I}_{ \pm}^{z z(1)}(\mathbf{k}, \omega) & =\perp_{ \pm}^{\mathscr{I}_{ \pm}^{z z(1)}}(\mathbf{k},-\omega) .
\end{aligned}
$$

The functions $\mathscr{I}_{ \pm}^{z z(\mathbf{1})}(\mathbf{k}, \omega)$ are given by

$$
\begin{aligned}
\mathscr{I}_{+}^{z z(1)}(\mathbf{k}, \omega)= & k^{2}\left(v^{(1)}+S^{(1)}-\mu^{(1)}-n^{(1)}\right) \\
& +k^{2} \epsilon_{k} \omega^{-2}\left(\epsilon_{k}-1\right)\left(\epsilon_{k} n^{(1)}+S^{(1)}-M_{2}^{(1)}-\mu^{(1)}\right) \\
& -k \epsilon_{k} \omega^{-2}\left[\omega \Lambda_{\mu}^{z(1)} \delta_{\mu}+\left(\epsilon_{k}-1\right) \Lambda_{\mu}^{z(1)} \beta_{\mu}\right] \\
& +k^{4} \omega^{-1} A^{(1)}-k^{2} \omega^{-2}\left[\left(\epsilon_{k}+1\right)\left(\omega^{2}-\epsilon_{k}{ }^{2}\right)+k^{2} \epsilon_{k}\right] F^{z z r(1)},
\end{aligned}
$$




$$
\begin{aligned}
\mathscr{F}_{-}^{z z(1)}(\mathbf{k}, \omega)= & k^{2} \omega^{-1}\left[\left(\epsilon_{k}^{2}-k^{2}\right) n^{\prime(1)}+\epsilon_{k}\left(S^{(1)}-M_{2}^{(1)}-\mu^{(1)}\right)\right] \\
& -k^{3} \omega^{-1}\left[\omega \Lambda_{\mu}^{z(1)} \delta_{\mu}+\left(\epsilon_{k}-1\right) \Lambda_{\mu}^{z(1)} \beta_{\mu}\right] \\
& +k^{2} A^{(1)}-k^{2} \omega^{-1}\left(\omega^{2}-\epsilon_{k}{ }^{2}+k^{2}\right) F^{z z r(1)} .
\end{aligned}
$$

Equations (3.41)-(3.45) complete our expansion to $O(g)$ of the one-particle Green's function. We note that from (3.41) $\mathscr{G}_{++}^{(1)}(\mathbf{k}, \omega)$ can be expressed in terms of $\mathscr{F}^{(1)}(\mathbf{k}, \omega)$.

It should be mentioned that in the present first-order perturbation expansion it has not been assumed that $\mathscr{G}, \mathscr{F}$, and other functions can be expanded in a power series in the small parameter $g$. We have merely grouped together terms contributing to first-order in $g$ and made no assumption about the nature of the higher approximations in $g$.

\section{First-Order Calculation}

We now use the perturbation expansion developed in Section 3C to calculate explicitly in the long-wavelength limit the response functions to the first order beyond the Bogoliubov approximation. We will see that the dielectric function formulation coupled with the use of the generalized Ward identities (2.34)-(2.36) displays the essential features of a Bose system at zero temperature and is also responsible for the relative simplicity of the present first-order calculation. In particular the calculations exhibit the sharing of singularitics by the zero-helicity response functions, the relative weights of the singularities, the contribution of the multi-phonon processes, and the satisfaction of various sum rules. To first order in $g$ the diagrams of interest are the one-loop diagrams, which are considered in Section 4A. The one-loop diagrams are then applied to the evaluation of the elementary excitation spectrum in Section $4 \mathrm{~B}$, the dynamic structure function in Section $4 C$, the associated structure functions and sum rules in Section $4 D$, and the one-particle spectral function in Section 4E.

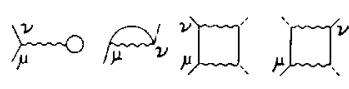

(a)

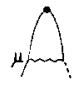

(b)

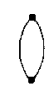

(c)

FIG. 6. One-loop diagrams for the regular functions: (a) the irreducible self energy $M_{\mu \nu}^{(1)}$; (b) the longitudinal-current vertex function $A^{z(1)}$; (c) the regular longitudinal-current response function $F^{z=r(1)}$. The solid line in a loop represents the Green's function $\mathscr{G}_{k t y}^{(0)}$ in the zeroth approximation and the heavy dot represents the longitudinal-current vertex $A_{u}^{z i 0}$ in the zeroth approximation. 


\section{A. One-Loop Diagrams}

From the discussion of Section 3B we know that to first order in $g$ the diagrams of interest are the one-loop diagrams. The one-loop diagrams for the regular functions $M_{\mu \nu}^{(1)}, \Lambda_{\mu}^{z(1)}, F^{z z r(1)}$ are shown in Fig. 6 . The dot represents the longitudinal current vertex which from (2.10) gives a factor of $\hat{\mathbf{k}} \cdot(\mathbf{p}+\mathbf{k} / 2) \beta_{\mu}$. The evaluation of the one-loop diagrams follows from the rules of standard zero-temperature perturbation theory [13]. We write

$$
\begin{aligned}
M_{\mu \nu}^{(1)}(k)= & i \int \frac{d^{4} p}{(2 \pi)^{4}}\left[\frac{1}{2} \mathscr{G}_{\lambda \lambda}^{(0)}(p) \delta_{\mu \nu}+\mathscr{G}_{\mu \nu}^{(0)}(p+k)\right] \\
& +i \int \frac{d^{4} p}{(2 \pi)^{4}}\left[\mathscr{G}_{\mu \nu}^{(0)}(p+k) \mathscr{G}_{\lambda \sigma}^{(0)}(p) \delta_{\lambda} \delta_{\sigma}\right. \\
& \left.+\mathscr{G}_{\mu \lambda}^{(0)}(p+k) \mathscr{G}_{-\sigma-\nu}^{(0)}(p) \delta_{\lambda} \delta_{\sigma}\right], \\
\Lambda_{\mu}^{z(\mathbf{1})}(k)= & \left.i \int \frac{d^{4} p}{(2 \pi)^{1}} \hat{\mathbf{k}} \cdot\left(\mathbf{p}+\frac{1}{2} \mathbf{k}\right) \beta_{\nu} \mathscr{G}_{\mu \nu}^{(0)}(p+k) \mathscr{G}_{\nu \lambda}^{(0)}(p) \delta_{\lambda}\right], \\
F^{z z r(1)}(k)= & \frac{1}{2} i \int \frac{d^{4} p}{(2 \pi)^{4}}\left(\hat{\mathbf{k}} \cdot\left(\mathbf{p}+\frac{1}{2} k\right)\right)^{2} \mathscr{G}_{\mu \nu}^{(0)}(p+k) \mathscr{G}_{\mu \nu}^{(0)}(p) \beta_{\nu} \beta_{\mu \nu},
\end{aligned}
$$

where the factor of $\frac{1}{2}$ in (4.3) comes from the symmetry of the diagram, the factor $i \int d^{4} p /(2 \pi)^{4}$ comes from the integration loop, $\delta_{\mu \nu}$ is the Kronecker delta function, $\delta_{\mu}=1, \beta_{\mu}=\operatorname{sgn}(\mu)$, and $p$ and $k$ stand for $(\mathbf{p}, \epsilon)$ and $(\mathbf{k}, \omega)$, respectively. The evaluation of the frequency integrals in (4.1)-(4.3) is straightforward and is given in Appendix A. We find the following one-loop integrals

$$
\begin{aligned}
& \frac{1}{2}\left(S^{(1)}+M_{2}^{(1)}-\mu^{(1)}\right)=\frac{1}{2} \int \frac{d^{3} p}{(2 \pi)^{3}} \lambda_{\mathbf{p}} \lambda_{\mathbf{p}+\mathbf{k}} Q^{+}, \\
& \frac{1}{2}\left(S^{(1)}-M_{2}^{(1)}-\mu^{(1)}\right)=\frac{1}{4} \int \frac{d^{3} p}{(2 \pi)^{3}}\left[\frac{1-\lambda_{\mathbf{p}}{ }^{2}}{\lambda_{\mathbf{p}}}+\left(\frac{\lambda_{\mathbf{p}}}{\lambda_{\mathbf{p}+\mathbf{k}}}+1\right) Q^{+}\right], \\
& A^{(1)}=\int \frac{d^{3} p}{(2 \pi)^{3}} \lambda_{\mathbf{p}} Q^{-}, \\
& A_{\mu}^{z(1)} \delta_{\mu}=\frac{1}{2} \int \frac{d^{3} p}{(2 \pi)^{3}}\left(\lambda_{\mathrm{p}}-\lambda_{\mathbf{p}+\mathbf{k}}\right)\left(\mathbf{p} \cdot \hat{\mathbf{k}}+\frac{1}{2} k\right) Q^{-}, \\
& \Lambda_{\mu}^{z(1)} \beta_{\mu}=\frac{1}{2} \int \frac{d^{3} p}{(2 \pi)^{3}}\left(\frac{\lambda_{\mathbf{p}}}{\lambda_{\mathbf{p}+\mathbf{k}}}-1\right)\left(\mathbf{p} \cdot \hat{\mathbf{k}}+\frac{1}{2} k\right) Q^{+}, \\
& F^{z z r(1)}=\frac{1}{8} \int \frac{d^{3} p}{(2 \pi)^{3}} \frac{\left(\lambda_{\mathbf{p}}-\lambda_{\mathbf{p}+\mathbf{k}}\right)^{2}}{\lambda_{\mathbf{p}} \lambda_{\mathbf{p}+\mathbf{k}}}\left(\mathbf{p} \cdot \hat{\mathbf{k}}+\frac{1}{2} k\right)^{2} Q^{+},
\end{aligned}
$$


where

$$
\begin{gathered}
Q^{ \pm}=\left(\omega-\omega_{\mathbf{p}+\mathbf{k}}^{(0)}-\omega_{\mathbf{p}}^{(0)}\right)^{-1} \mp\left(\omega+\omega_{\mathbf{p}+\mathbf{k}}^{(0)}+\omega_{\mathbf{p}}^{(0)}\right)^{-1}, \\
\lambda_{\mathbf{p}}=\epsilon_{\mathbf{p}} / \omega_{\mathbf{p}}^{(0)}=\frac{1}{2} p\left(1+\frac{1}{4} p^{2}\right)^{-1 / 2} .
\end{gathered}
$$

Whenever a three-dimensional (or less) integral over $p$ is indicated as in (4.4)-(4.11), we interpret $p=|\mathbf{p}|$, not to be confused with the four-notation in (4.1) (4.3).

Other integrals that are necessary include $n^{(1)}$ and $v^{(1)}$. The number of particles not in the condensate is obtained from the Green's function $\mathscr{G}_{++}$by making the one-loop sum:

$$
\begin{aligned}
n^{\prime(1)} & =-i \int \frac{d^{4} p}{(2 \pi)^{4}} \mathscr{G}_{++}^{(0)}(p) e^{\prime 0+} \\
& =\frac{1}{4} \int \frac{d^{3} p}{(2 \pi)^{3}} \frac{\left(1-\lambda_{p}\right)^{2}}{\lambda_{p}}=\frac{1}{3 \pi^{2}} .
\end{aligned}
$$

The quantity $v^{(1)}$ can be obtained from the perturbation expansion for the zeroenergy vacuum scattering amplitude $g$ in powers of $v$

$$
g=4 \pi a=v+\int \frac{d^{3} p}{(2 \pi)^{3}} v \frac{1}{\epsilon_{p}-\epsilon_{p}} v+O\left(v^{3}\right),
$$

where the initial and final energies are taken to be zero. Rearranging (4.13), we find

$$
\begin{aligned}
v & =g+g^{2} v^{(1)}+O\left(g^{3}\right) \\
\iota^{(1)} & =\int \frac{d^{3} p}{(2 \pi)^{3}} \frac{1}{2 \epsilon_{p}} .
\end{aligned}
$$

The large- $p$ divergence of $v^{(1)}$ comes from the point nature of our model interaction and will be cancelled by a similar large- $p$ divergence in $S^{(1)}+M_{2}^{(1)}-\mu^{(1)}$ and $S^{(1)}-\mu^{(1)}$.

\section{B. Elementary Excitation Spectrum}

The elementary excitation spectrum of a Bose system is determined by the discrete pole that is shared by all the zero-helicity response functions, e.g., $\mathscr{G}(\mathbf{k}, \omega)$, $\mathscr{F}(\mathbf{k}, \omega), \mathscr{F}^{z z}(\mathbf{k}, \omega)$, etc. The elementary excitation is thus a discrete zero-helicity excited state of the system which we refer to as a "phonon" in the long-wavelength limit. From (2.17) and (2.45), we see that a zero in the dielectric function $\epsilon(\mathbf{k}, \omega)$ produces such a common pole in $\mathscr{F}$ and $\mathscr{G}$. It might be thought from (2.45) that a pole of $G$, i.e., a zero of $D$, would also produce a pole in $\mathscr{G}$. However, we see from (2.17) and (2.23) that a pole of $G$ is also a pole of $\epsilon(\mathbf{k}, \omega)$ and thus is cancelled. 
We proceed to solve the equation $\epsilon(\mathbf{k}, \omega)=0$ for the elementary excitation spectrum $\omega_{k}$. As pointed out earlier (see (3.36)), it is useful to express $\epsilon$ in terms of the current correlation function $F^{z z}$ so that the equation $\epsilon=0$ becomes

$$
\omega_{k}^{2} / k^{2}=(v / g)\left[1+g F^{z z}\left(\mathbf{k}, \omega_{k}\right)\right]
$$

We develop a perturbation expansion for $\omega_{k}$ by expanding all terms in (4.15) to first order in $g$

$$
\begin{aligned}
\omega_{k}^{2} & =\omega_{k}^{2(0)}+g \omega_{k}^{2(1)}+O\left(g^{2}\right), \\
g F^{z z} & =F^{z z(0)}+g\left[F^{z z(\mathbf{1})}+\omega_{k}^{2(1)} \partial F^{z z(0)} / \partial \omega^{2}\right]+O\left(g^{2}\right) .
\end{aligned}
$$

The expansion of $v / g$ is given in (3.26). All quantities on the right side of (4.17) are evaluated at $\omega_{k}^{2(0)}$. In zeroth order we find $F^{z z(0)}=\epsilon_{k}^{2}\left(\omega^{2}-\epsilon_{k}^{2}\right)^{-1}$ from (3.7) and from (4.15)-(4.17) and (3.26) the result $\omega_{k}^{2(0)}=k^{2}\left(1+k^{2} / 4\right)$, which is in agreement with (3.10). In first order we find from (4.15)-(4.17), (3.7), and (3.32a) that

$$
\omega_{k}^{2(1)}=\mathscr{N}^{z z(1)}\left(\mathbf{k}, \omega_{k}^{(0)}\right) / \omega_{k}^{2(0)},
$$

where $\mathscr{N}^{z z(1)}(\mathbf{k}, \omega)$ is given by (3.32b). It is convenient to combine (4.18) with (3.32b) in the following manner

$$
\begin{aligned}
\omega_{k}^{2(1)} / k^{2}= & v^{(1)}+\frac{1}{2}\left(S^{(1)}+M_{2}^{(1)}-\mu^{(1)}\right)+\left(k^{4} / 4 \omega_{k}^{2(0)}\right) \frac{1}{2}\left(S^{(1)}-M_{2}^{(1)}-\mu^{(1)}\right) \\
& +\left(k^{2} / \omega_{k}^{2(0)}\right)\left[k^{-1} \omega_{k}^{(0)} \Lambda_{\mu}^{z(1)} \delta_{k 2}+\frac{1}{2} k \Lambda_{\mu}^{z(1)} \beta_{\mu}-\frac{1}{4} k^{2} n^{\prime(1)}\right. \\
& \left.+\frac{1}{2} \omega_{k}^{(0)} A^{(1)}+F^{z z r(1)}\right] .
\end{aligned}
$$

The above perturbation expansion for $\omega_{k}$ is not meaningful unless $\omega_{k}$ is away from the singularities of $\epsilon(\mathbf{k}, \omega)$. As discussed above, $\epsilon(\mathbf{k}, \omega)$ has a pole located at the pole of $G(\mathbf{k}, \omega)$, which, from (3.9), is seen to be located at the free particle energy $\epsilon_{k}$ in the zeroth order calculation. In the long-wavelength limit this singularity in $\epsilon(\mathbf{k}, \omega)$ is far away from $\omega_{k}$ and causes no difficulty. Branch cuts in $\epsilon$ comes from the branch cuts of the regular functions $M_{\mu \nu}, \Lambda_{\mu}{ }^{z}$, and $F^{z z r}$, which arise from diagrams with an intermediate state with at least two excitations. Since to first order in $g$ there are no bound states between two excitations, the regular functions do not have a pole in addition to the branch cut starting at the two excitation threshold (see Section 4C). The branch cuts however do not cause any difficulty as we can analylically continue $\epsilon(\mathbf{k}, \omega)$ across the branch cut and solve (4.15) for a complex $\omega_{k}$.

We consider here only the real part of the excitation spectrum which we denote as simply $\omega_{k}$. The calculation of the imaginary part, denoted by $\operatorname{Im} \omega_{k}$, is done in 
Appendix C. We need to evaluate the regular functions (4.4)-(4.9) at $\omega=\omega_{k}^{(0)}$ with $Q^{ \pm}$given by (see (4.10))

$$
Q^{ \pm}=\left(\omega_{\mathbf{k}}^{(0)}-\omega_{\mathbf{p}+\mathbf{k}}^{(\mathbf{0})}-\omega_{\mathbf{p}}^{(0)}\right)^{-1} \mp\left(\omega_{\mathbf{k}}^{(0)}+\omega_{\mathbf{p}+\mathbf{k}}^{(0)}+\omega_{\mathbf{p}}^{(0)}\right)^{-1} .
$$

Since we are interested in the long-wavelength limit of $\omega_{k}$, we expand the integrals (4.4)-(4.9) in powers of $k$. A summary of all the relevant $k$-expansions is given in Appendix B. As a simple example consider the expansion of the function $A^{(1)}$

$$
A^{(1)}=a_{1} k+a_{2} k^{2}+a_{3} k^{3}+\cdots,
$$

where

$$
\begin{aligned}
& a_{1}=-\frac{1}{2} \int \frac{d^{3} p}{(2 \pi)^{3}} \lambda_{p} / \omega_{p}^{2(0)}=-\frac{1}{2 \pi^{2}}, \\
& a_{3}=-\frac{1}{2} \int \frac{d^{3} p}{(2 \pi)^{3}} \lambda_{p}\left(5(\hat{\mathbf{k}} \cdot \hat{\mathbf{p}})^{2}-1\right) / 4 p^{2} \omega_{p}^{2(0)} .
\end{aligned}
$$

It is easy to see that $a_{2}=0$ from symmetry considerations. The integral $a_{3}$ converges for large $p$ but has a logarithmic singularity for small $p$. We cut off the small-p logarithmic singularity at $k$ and obtain $a_{3}=-\left(1 / 48 \pi^{2}\right) \ln (1 / k)$ plus a nonsingular term which is not amenable to calculation by this procedure. In this manner the integrals (4.4)-(4.9) are evaluated to be

$$
\begin{aligned}
v^{(1)}+\frac{1}{2}\left(S^{(1)}+M_{2}^{(1)}-\mu^{(2)}\right) & =\frac{2}{\pi^{2}}+\frac{1}{36 \pi^{2}} k^{2}-\frac{1}{480 \pi^{2}} k^{4} \ln (1 / k)+O\left(k^{4}\right), \\
\frac{1}{2}\left(S^{(1)}-M_{2}^{(1)}-\mu^{(1)}\right) & =-\frac{1}{16 \pi^{2}} k^{2} \ln (1 / k)+O\left(k^{2}\right), \\
A^{(1)} & =-\frac{1}{2 \pi^{2}} k-\frac{1}{48 \pi^{2}} k^{3} \ln (1 / k)+O\left(k^{3}\right), \\
\Lambda_{\mu}^{z(1)} \delta_{\mu} & =\frac{1}{36 \pi^{2}} k^{2}+\frac{1}{480 \pi^{2}} k^{4} \ln (1 / k)+O\left(k^{4}\right), \\
\Lambda_{\mu}^{z(1)} \beta_{\mu} & =\frac{1}{3 \pi^{2}} k+\frac{1}{48 \pi^{2}} k^{3} \ln (1 / k)+O\left(k^{3}\right), \\
F^{z z r(1)} & =-\frac{1}{60 \pi^{2}} k^{2}-\frac{1}{320 \pi^{2}} k^{4} \ln (1 / k)+O\left(k^{4}\right) .
\end{aligned}
$$

In writing down (4.21) we have used the fact that from (4.14)

$$
v^{(1)}=\frac{2}{\pi^{2}}-I_{0},
$$


where

$$
I_{0}=-\frac{1}{2} \int \frac{d^{3} p}{(2 \pi)^{3}} \frac{\lambda_{p}{ }^{2}}{\omega_{p}}
$$

is divergent for large $p$. This large- $p$ divergence arises from the point nature of the model interaction and appears also in the integral (4.4) for $\frac{1}{2}\left(S^{(1)}+M_{\mathbf{2}}^{(\mathbf{1})}-\mu^{(\mathbf{1})}\right)$. However these large- $p$ divergences exactly cancel as shown in the first line of (4.21).

If we substitute (4.12) and (4.21) into (4.18), we find that up to $O(g)$ the form of $\omega_{k}$ for small $k$ is

$$
\omega_{k} / k=c_{0}+c_{2} k^{2}+c_{L 4} k^{4} \ln (1 / k)+O\left(k^{4}\right),
$$

where

$$
\begin{gathered}
c_{0}=1+\frac{g}{\pi^{2}}+O\left(g^{2}\right), \\
c_{2}=\frac{1}{8}-\frac{17 g}{90 \pi^{2}}+O\left(g^{2}\right), \\
c_{L 4}=-\frac{3 g}{320 \pi^{2}}+O\left(g^{2}\right) .
\end{gathered}
$$

The form of (4.24) demonstrates the main result of this subsection, that is, to first order in $g$ the real part of the elementary excitation spectrum is not an analytic function of the wavevector $k$ in the long wavelength limit. The value of the sound speed $c_{0}$ given in (4.24b) agrees with earlier calculations [4-6, 8].

In many previous calculations $[4-6]$ the excitation spectrum is determined from the zero of the denominator $\mathscr{D}$ of the Green's function (see (2.37)-(2.39))

$$
\mathscr{D}=\left(\omega_{k}-\mathscr{A}\right)^{2}-\left(\epsilon_{k}-\mu+\mathscr{S}-\mathscr{M}_{2}\right)\left(\epsilon_{k}-\mu+\mathscr{S}+\mathscr{M}_{2}\right)=0 .
$$

Recall that the script letters indicate that the reducible self-energy diagrams are also included in addition to the irreducible diagrams evaluated in this section. In the present method we determine $\omega_{k}$ from the zero of the dielectric function written in the form (4.15). If we compare (4.15) and (4.25) we note the following advantages of the present method for calculations of $\omega_{k}$ in the long-wavelength limit. First, the presence of the factor $\omega^{2} / k^{2}$ in (4.15) allows us to obtain the phonon speed $c_{0}$ straightforwardly by taking the $k \rightarrow 0$ limit of $F^{z z}$ without any expansion of the integrals. Second, the two longitudinal current vertices of $F^{z z}$ give rise to two powers of momentum and make the small momentum part of various integrals better behaved. Spurious divergences are thus eliminated. Third, the zeroth (Bogoliubov) order term, $n v / m$, is explicit in (4.15) and enters with the input 
parameter $n$ instead of $n_{0}$. Thus there is no need for the substitution $n_{0}=n-n^{\prime}$ and the sum rules are automatically satisfied (see next section).

Note that we did not have to calculate explicitly the single-particle self-energy functions $\mathscr{M}_{\mu \nu}$ but have instead calculated the regular functions $M_{\mu \nu}, \Lambda_{\mu \nu}{ }^{z}$, and $F^{z z r}$. The explicit form of $\mathscr{M}_{\mu \nu}^{(1)}$ can be found by using (2.41), (3.37), (3.39), and (4.4)-(4.49).

\section{Dynamic Structure Function}

The dynamic structure function $\mathscr{H}(\mathbf{k}, \omega)$ represents the maximum information that can be obtained about the properties of a system from an experiment using a density probe, e.g., a neutron scattering experiment. $\mathscr{T}(\mathbf{k}, \omega)$ is defined as the positive frequency spectral function of $\mathscr{F}$

$$
\mathscr{S}(\mathbf{k}, \omega)=-\frac{1}{\pi} \operatorname{Im} \mathscr{\mathscr { F }}(\mathbf{k}, \omega), \quad \omega \geqslant 0 .
$$

$\mathscr{S}(\mathbf{k}, \omega)=0$ for $\omega<0$ at zero temperature. The spectral function of $\mathscr{F}$ for all $\omega$ is simply

$$
\mathscr{P}(\mathbf{k}, \omega)-\mathscr{F}(\mathbf{k},-\omega)=-\frac{1}{\pi} \operatorname{Im} \mathscr{\mathscr { F }}(\mathbf{k}, \omega),
$$

which can be interpreted as the fluctuation-dissipation theorem.

In zeroth order $\mathscr{P}^{(0)}$ is found from the relation (4.26) and the result (3.11) for $\mathscr{F}^{(0)}$ to have the form

$$
\begin{aligned}
\mathscr{P}^{(0)}(\mathbf{k}, \omega) & =Z^{(0)}(k) \delta\left(\omega-\omega_{k}^{(0)}\right) \\
Z^{(0)}(k) & =k^{2} / 2 \omega_{k}^{(0)} .
\end{aligned}
$$

The first-order correction $\mathscr{S}^{(1)}$ is found from (3.31) and (4.26) to be

$$
\mathscr{S}^{(1)}(\mathbf{k}, \omega)=-\frac{k^{2}}{\pi} \operatorname{Im}\left[\omega^{-2}\left(\omega^{2}-\omega_{k}^{2(0)}\right)^{-2} \mathscr{N}^{z z(1)}(\mathbf{k}, \omega)\right],
$$

where $\mathscr{N}^{z z(1)}(\mathbf{k}, w)$ is given by (3.32). From (4.29) we see that there are three terms, $\omega^{-2},\left(\omega^{2}-\omega_{k}^{2(0)}\right)^{-2}$, and $\mathscr{N}^{z z(1)}(\mathbf{k}, \omega)$, of which the imaginary part must be taken to determine $\mathscr{S}^{(1)}$. Recall that $\mathscr{F}$ is a response function so that $\omega$ should be interpreted as $\omega+i 0+$. We now show that in (4.29) the double pole at $\omega=0$ is cancelled. The identity (2.31) implies that $\omega^{2} F^{(1)}=k^{2} F^{z z(1)}$ and hence $\mathscr{N}^{z z(1)}(\mathbf{k}, \omega)=\omega^{2} k^{-2} \mathscr{N}^{(1)}(\mathbf{k}, \omega)$, where $\mathscr{N}^{(1)}(\mathbf{k}, \omega)$ is given by (3.33) and (3.34). 
It is easy to show that $\mathscr{N}^{(\mathbf{1})}(\mathbf{k}, 0)$ is a constant, so that for small $\omega, \mathscr{N}^{z z(1)}(\mathbf{k}, \omega) \propto \omega^{2}$ and the double pole at $\omega=0$ in (4.29) is cancelled. We thus have

$$
\begin{aligned}
\mathscr{P}^{(\mathbf{1})}(\mathbf{k}, \omega)- & -k^{2} \omega^{-2} \mathscr{N}^{z z(1)}(\mathbf{k}, \omega)\left(4 \omega_{k}^{3(0)}\right)^{-1}\left[\delta\left(\omega-\omega_{k}^{(0)}\right)+\omega_{k}^{(0)} \delta^{\prime}\left(\omega \quad \omega_{k}^{(0)}\right)\right] \\
& -k^{2} \omega^{-2} \operatorname{Re}\left(\omega^{2}-\omega_{k}^{2(0)}\right)^{-2}(1 / \pi) \operatorname{Im} \mathscr{N}^{z z(\mathbf{1})}(\mathbf{k}, \omega),
\end{aligned}
$$

where the delta functions arise from $\operatorname{Im}\left(\omega^{2}-\omega_{k}^{2(0)}\right)^{-2}$.

In general discussions [14] of the form of $\mathscr{S}(\mathbf{k}, \omega)$, it is common to separate it into two terms. However in discussing the interpretation of (4.30), we will see that it is necessary to make a somewhat different separation. We first note that in zeroth order $\mathscr{P}^{(0)}(\mathbf{k}, \omega)$ exhausts the $f$ sum rule

$$
\int_{0}^{\infty} d \omega \omega g \mathscr{S}(\mathbf{k}, \omega)=k^{2} / 2
$$

which is derived in Appendix D. We are thus led to write $\mathscr{S}^{(1)}(\mathbf{k}, \omega)$ as a sum of three terms

$$
\mathscr{S}^{(1)}(\mathbf{k}, \omega)=\mathscr{S}_{F}^{(1)}(\mathbf{k}, \omega)+\mathscr{S}_{B}^{(1)}(\mathbf{k}, \omega)+X^{(1)}(\mathbf{k}, \omega),
$$

where the one-phonon part $\mathscr{S}_{F}^{(1)}(\mathbf{k}, \omega)$ is defined to exhaust the $f$ sum rule

$$
\begin{aligned}
g \mathscr{S}_{F}(\mathbf{k}, \omega) & =Z_{F}(k) \delta\left(\omega-\omega_{k}\right), \\
Z_{F}(k) & \equiv k^{2} / 2 \omega_{k} .
\end{aligned}
$$

From (4.33) and (4.28), we see that $\mathscr{S}^{(0)}(\mathbf{k}, \omega)=\mathscr{S}_{F}^{(0)}(\mathbf{k}, \omega)$, which expresses the well known fact that there are no multiexcitation processes in zeroth order. Expanding (4.33) to first order, we get

$$
\mathscr{S}_{F}^{(1)}(\mathbf{k}, \omega)=Z_{F}^{(1)}(k)\left[\delta\left(\omega-\omega_{k}^{(0)}\right)+\omega_{k}^{(0)} \delta^{\prime}\left(\omega-\omega_{k}^{(0)}\right],\right.
$$

where

$$
\begin{gathered}
Z_{F}^{(1)}(k)=-\frac{1}{2} Z_{F}^{(0)}(k) \omega_{k}^{2(1)} / \omega_{k}^{2(0)} \\
=f_{1} k+f_{3} k^{3}+f_{L 5} k^{5} \ln (1 / k)+O\left(k^{5}\right), \\
f_{1}=-\frac{1}{2} \pi^{-2}, \\
f_{3}=(79 / 360) \pi^{-2} \\
f_{L 5}=(3 / 640) \pi^{-2} .
\end{gathered}
$$

The numerical coefficients $f_{1}, f_{3}, f_{L 5}$ are obtained from (3.10), (4.16), (4.24a), and (4.25a). We note that the one-phonon intensity function $Z_{F}(k)$ is not an analytic function of $k$. 
If we compare (4.30) and (4.34) we see that there is still a remaining deltafunction part

$$
\begin{aligned}
\mathscr{S}_{B}^{(1)}(\mathbf{k}, \omega) & =Y^{(1)}(\mathbf{k}, \omega) \delta^{\prime}\left(\omega-\omega_{k}^{(0)}\right), \\
Y^{(1)}(\mathbf{k}, \omega) & =-\left(4 \omega_{k}^{2(0)}\right)^{-1}\left[k^{2} \omega^{-2} \mathscr{N}^{z z(1)}(\mathbf{k}, \omega)-k^{2} \omega_{k}^{2(1)}\right] .
\end{aligned}
$$

$\mathscr{S}_{B}$ will be referred to as the backflow contribution for reasons which will be apparent shortly. From (4.18) we see that $Y^{(1)}\left(\mathbf{k}, \omega_{k}^{(0)}\right)=0$. If we expand $Y^{(1)}(\mathbf{k}, \omega)$ about $\omega_{k}^{(0)}$, we have

$$
Y^{(\mathbf{1})}(\mathbf{k}, \omega)=-Y^{(\mathbf{1})}(k) \cdot\left(\omega-\omega_{k}^{(0)}\right)+O\left(\left(\omega-\omega_{k}^{(0)}\right)^{2}\right),
$$

where

$$
\begin{aligned}
Y^{(1)}(k) & =\frac{k^{2}}{4 \omega_{k}^{2(0)}} \frac{d}{d \omega}\left[\omega^{-2} \mathcal{N}^{z z(1)}(\mathbf{k}, \omega)\right]_{\omega_{k}^{(n)}} \\
& =y_{3} k^{3}+y_{L 5} k^{5} \ln (1 / k)+O\left(k^{5}\right)
\end{aligned}
$$

and

$$
\begin{aligned}
y_{3} & =-7 /\left(1440 \pi^{2}\right), \\
y_{L 5} & =-3 /\left(640 \pi^{2}\right) .
\end{aligned}
$$

The numerical evaluation of $y_{3}$ and $y_{L 5}$ follows straightforwardly from (3.32), (4.4)-(4.49), and (4.38a) using the same procedure as in the calculation of $\omega_{k}$. If we substitute (4.37) into (4.36a) and note that we are only interested in the $\omega$-integral of $\mathscr{P}(\mathbf{k}, \omega)$ multiplied by a smooth function, we find the form

$$
\mathscr{S}_{B}^{(1)}(\mathbf{k}, \omega)=Y^{(1)}(k) \delta\left(\omega-\omega_{k}^{(0)}\right),
$$

where we have used the symbolic identity $x \delta^{\prime}(x)=-\delta(x)$. Further discussion of $Y^{(\mathbf{1})}(k)$ and its evaluation in (4.38) can be found in Appendix B following Eq. (B.3). Note that $\mathscr{S}_{B}^{(1)}$ cannot be grouped immediately with $\mathscr{S}_{F}^{(1)}$ since the delta-function in (4.39) is at $\omega_{k}^{(0)}$ and not at $\omega_{k}^{(0)}+\frac{1}{2} g \omega^{2(1)} / \omega_{k}^{(0)}$ as in (4.34).

The remaining part of $\mathscr{S}^{(1)}(\mathbf{k}, \omega)$ is the incoherent two-phonon background contribution given by

$$
X^{(1)}(\mathbf{k}, \omega)=-k^{2} \omega^{-2} \operatorname{Re}\left(\omega^{2}-\omega_{k}^{2(0)}\right)^{-2} \pi^{-1} \operatorname{Im} \mathscr{N}^{z z(1)}(\mathbf{k}, \omega) .
$$

We now interpret the three contributions (4.34), (4.39), and (4.40) to $\mathscr{S}^{(1)}(\mathbf{k}, \omega)$ in terms of the first-order physical processes shown in Fig. 7. The structure of $\mathscr{F}(1)$, (3.31), is represented by Fig. 7a, where the one-loop part denotes $\mathscr{N}^{z z(1)}$. Taking the imaginary part of $\mathscr{F}^{(1)}$ to obtain $\mathscr{S}^{(1)}$ corresponds to cutting the diagram in Fig. 7a in all possible ways as denoted by the dashed lines. The term $\mathscr{S}_{F}^{(1)}$, given by (4.34) 


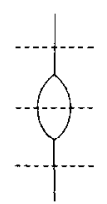

(a)

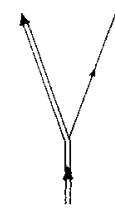

(0)

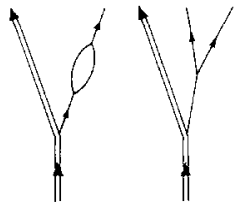

(c)

(d)

Fig. 7. First-order Multi-Excitation Processes. The structure of the vertices is ignored. (a) The density response function $\mathscr{F}(\mathbf{k}, \omega)$. Solid lines represent excitations and the dashed lines denote cuts needed to obtain the imaginary part, $\mathscr{H}(\mathbf{k}, \omega)$. (b)-(d) The production of elementary excitations (single line) by particle probe (double line). Excitations represented by directed lines are on the energy shell. Figures (b), (c), and (d) correspond to the one-phonon, backflow, and background contributions respectively.

arises from the imaginary part of the single pole corresponding to either the upper or the lower cut in Fig. 7a and represents (Fig. 7b) the production of a real phonon to $O(g)$. The term $\mathscr{P}_{B}^{(1)},(4.39)$, arises from the imaginary part of the double pole due to the coalescence of the single poles of the two isolated lines of Fig. 7 a. $\mathscr{S}_{B}^{(1)}$ can be represented by Fig. 7c, the production of a real phonon in zeroth order that decays in $O(g)$ into two virtual phonons which in turn recombine into a real phonon. The real phonon can be pictured as being surrounded by a cloud of two virtual phonons. As the real phonon moves, it drags the cloud of virtual phonons along with it, which is equivalent to the backflow picture [14]. Lastly $X^{(1)},(4.40)$, arises from the imaginary part of the two excitations in $\mathcal{N}^{z z(1)}$ and can be interpreted as (Fig. 7 d) the production of a virtual phonon that subsequently decays into two real phonons. $\mathscr{S}_{F}^{(1)}, \mathscr{S}_{B}^{(1)}$, and $X^{(1)}$ will be referred to as the one-phonon, backflow, and background terms respectively.

The two coherent terms (4.34) and (4.39) are proportional to a delta function by definition, and only the background term $X^{(1)}(\mathbf{k}, \omega)$ given by (4.40), has interesting structure. We first examine the threshold behavior of $X^{(1)}(\mathbf{k}, \omega)$. From (3.32) and (4.4)-(4.49) it is easy to see that the integrand of $X^{(1)}$ is proportional to the imaginary part of $Q^{ \pm}$which is given by (see (4.10))

$$
-\frac{1}{\pi} \operatorname{Im} Q^{ \pm}=\delta\left(\omega-\omega_{\mathbf{p}+\mathbf{k}}^{(0)}-\omega_{\mathbf{p}}^{(0)}\right) \mp \delta\left(\omega+\omega_{\mathbf{p}+\mathbf{k}}^{(0)}+\omega_{\mathbf{p}}^{(0)}\right) .
$$

Since $X^{(1)}$ is nonzero only for positive $\omega$, the second term in (4.41) does not enter into the calculation of $X^{(1)}$, and we see that the threshold for the production of two excitations is simply

$$
\omega_{\mathrm{thr}}^{(0)}=2 \omega_{k / 2}^{(0)}=k\left(1+k^{2} / 16\right)^{1 / 2} .
$$

In other words, $X^{(1)}(\mathbf{k}, \omega)$ is zero for $\omega<\omega_{\mathrm{thr}}^{(0)}$. Note that for nonzero $k$, 
$\omega_{\mathrm{tnr}}^{(0)}<\omega_{k}^{(0)}$ and thus to $O(g)$ the elementary excitation spectrum is unstable. To determine the behavior of $X^{(1)}$ near threshold, we rewrite the first term in (4.41) in symmetric form and expand it about $\omega_{\mathrm{thr}}^{(0)}$

$$
\delta\left(\omega_{\mathbf{k} / 2+\mathbf{p}}^{(0)}+\omega_{\mathbf{k} / 2-\mathbf{p}}^{(0)}-\omega\right) \approx \delta\left(-\omega+2 \omega_{k / 2}^{(0)}+p^{2} / m_{k}\right),
$$

where

$$
\begin{aligned}
1 / m_{k} & =\left(d^{2} \omega_{p}^{(0)} / d p^{2}\right)_{p=k / 2} \\
& =\frac{3}{8} \frac{k^{2}}{\omega_{\mathrm{thr}}^{(0)}}\left[1-k^{4} / 48 \omega_{\mathrm{thr}}^{2(0)}\right]
\end{aligned}
$$

measures the curvature of the spectrum at $k / 2$. The behavior of the incoherent background $X^{(1)}(\mathbf{k}, \omega)$ near threshold can now be explicitly evaluated using (4.43) and we obtain

$$
X^{(1)}(\mathbf{k}, \omega)=\left(3 / 8 \pi^{2}\right) m_{k}^{3 / 2}\left(\omega-\omega_{t h r}^{(0)}\right)^{1 / 2}\left[1+O\left(\left(\omega-\omega_{t h r}^{(0)}\right) / k^{3}\right)\right] .
$$

We see from (4.45) that the branch point at the two-excitation threshold for $X^{(\mathbf{1})}$, as well as the regular functions (4.4)-(4.9), is a square root singularity.

We can establish the small $k$ behavior of $X^{(1)}(\mathbf{k}, \omega)$ by using arguments similar to those following (4.29) to show that for fixed $\omega, \mathscr{N}^{z z(1)}(\mathbf{k}, \omega) \propto k^{2}$ for small $k$. From (4.40) we see that for a fixed $\omega>\omega_{\text {thr }}^{(0)}$,

$$
\lim _{k \rightarrow 0} X^{(1)}(\mathbf{k}, \omega) \propto k^{4} .
$$

We can also determine the behavior of $X^{(1)}(\mathbf{k}, \omega)$ in the limit $\omega \gg k \omega^{1 / 2} \gg 1$ by writing the first term in (4.41) as

$$
\delta\left(\omega-\omega_{\mathbf{p}+\mathbf{k}}^{(0)}-\omega_{\mathbf{p}}^{(0)}\right) \approx \delta\left(\omega-p^{2}\right) .
$$

Again the $p$-integrals can be done simply and we find that the large $\omega$ behavior of $X^{(1)}(\mathbf{k}, \omega)$ is given by

$$
X^{(1)}(\mathbf{k}, \omega)=\left(7 / 120 \pi^{2}\right) \omega^{-3 / 2}\left(k^{2} / \omega\right)^{2}\left[1+O\left(k^{2} / \omega\right)\right] .
$$

The corrections to the large $\omega$ behavior of $X^{(1)}$ are order $k^{2} / \omega$, which can be understood by the following argument. The particle probe produces a highfrequency low-momentum virtual-excitation which decays into two excitations each of which must have approximately the same high frequency to conserve energy and momentum. Thus the two excitations are on the free-particle portion of the elementary excitation spectrum and

$$
\delta\left(\omega-\omega_{\mathbf{p}+\mathbf{k}}^{(0)}-\omega_{\mathbf{p}}^{(0)}\right) \approx \delta\left(\omega-p^{2}\left(1+u x^{1 / 2}+\frac{1}{2} x\right)\right),
$$


where $x=k^{2} / \omega$ and $u$ is the cosine of the angle between $\mathbf{p}$ and $\mathbf{k}$. If we integrate over $\mathbf{p}$, we are then left with a function of $x$. Equation (4.48) displays the leading dependence of such a function in the limit $1 \gg x^{1 / 2} \gg \omega^{-1}$.

\section{Structure Functions and Sum Rules}

Information concerning the dynamic structure function is frequently conveyed in terms of the structure functions, $\mathscr{S}_{m}(k)$, which are defined as the $m$ th frequency moment of $\mathscr{S}(\mathbf{k}, \omega)$

$$
g \mathscr{S}_{m}(k)=\int_{0}^{\infty} d \omega \omega^{m} g \mathscr{P}(\mathbf{k}, \omega)
$$

These structure functions are useful because they satisfy certain sum rules, e.g., (4.31). $\mathscr{S}_{0}(k)$, which is usually referred to as the static structure function, can be measured by $X$-ray scattering.

The separation (4.32) of $\mathscr{S}^{(1)}(\mathbf{k}, \omega)$ was useful since each term could be interpreted in terms of a physical process. However in discussing the moment functions, it is more convenient to combine $\mathscr{S}^{(0)}(\mathbf{k}, \omega)$ and $g_{\mathscr{S}} \mathscr{P}^{(\mathbf{1})}(\mathbf{k}, \omega)$ into one expression for $g \mathscr{S}(\mathbf{k}, \omega)$ as follows

$$
\begin{aligned}
g \mathscr{S}(\mathbf{k}, \omega)= & Z^{(0)}(k) \delta\left(\omega-\omega_{k}^{(0)}-g \omega_{k}^{(1)}\right) \\
& +g Z^{(1)}(k) \delta\left(\omega-\omega_{k}^{(0)}\right)+g X^{(1)}(k, \omega),
\end{aligned}
$$

where $Z^{(0)}(k)=Z_{F}^{(0)}(k), Z^{(1)}(k)=Z_{F}^{(0)}(k)+Y^{(1)}(k)$, and $\omega_{k}^{(1)}=\omega_{k}^{2(1)} / 2 \omega_{k}^{(0)}$. The first-order contribution may now be interpreted in terms of a backflow $Y^{(1)}$ and background $X^{(1)}$ contribution in addition to a shift in the spectrum $\omega_{k}$.

If we substitute (4.50) into (4.49), we can write the structure functions as

$$
g \mathscr{S}_{m}(k)=\left(\omega_{k}^{(0)}+g \omega_{k}^{(1)}\right)^{m} Z^{(0)}(k)+g \omega_{k}^{(0) m} Z^{(1)}(k)+g X_{m}^{(1)}(k),
$$

where $\omega_{k}=\omega_{k}^{(0)}+g \omega_{k}^{(1)}$. If we express (4.5la) in terms of the $c$-coefficients given in (4.24), we have

$$
\begin{aligned}
g \mathscr{S}_{m}(k)= & \left(\frac{1}{2} c_{0}{ }^{m}+z_{1}\right) k^{m+1}+\left(\frac{1}{2} m c_{2} c_{0}^{m-1}-\frac{1}{16} c_{0}{ }^{m}+\frac{1}{8} m z_{1}+z_{3}\right) k^{m+3} \\
& +\left(\frac{1}{2} m c_{L 4} c_{0}^{m-1}\right) k^{m+5} \ln (1 / k)+g X_{m}^{(1)}(k) \\
& +O\left(k^{m+5}\right)+O\left(g^{2} k^{m+1}\right),
\end{aligned}
$$

where the $z$-coefficients are obtained by summing (4.35) and (4.38),

$$
\begin{aligned}
g Z^{(1)}(k) & =z_{1} k+z_{3} k^{3}+O\left(k^{5}\right), \\
z_{1} & =-\left(1 / 2 \pi^{2}\right) g, \\
z_{3} & =\left(103 / 480 \pi^{2}\right) g .
\end{aligned}
$$


Note that (4.52) does not have any logarithmic terms through $O\left(k^{5}\right)$. The background contribution defined by

$$
X_{m}^{(1)}(k)=\int_{0}^{\infty} d \omega \omega^{m} X^{(1)}(\mathbf{k}, \omega)
$$

is evaluated in the small- $k$ limit from (3.32), (4.40) and (4.53a) by performing first the $\omega$ integral with the aid of (4.41). The leading $k$-dependences are thus found to be (see Appendix B)

$$
\begin{aligned}
& X_{-1}^{(1)}(k)=\left(7 / 7680 \pi^{2}\right) k^{4} \ln (1 / k)+O\left(k^{4}\right), \\
& X_{0}^{(1)}(k)=(7 / 10240 \pi) k^{4}+\cdots, \\
& X_{1}^{(1)}(k)=\left(7 / 1440 \pi^{2}\right) k^{4}+\left(3 / 640 \pi^{2}\right) k^{6} \ln (1 / k)+O\left(k^{6}\right), \\
& X_{2}^{(1)}(k)=(7 / 480 \pi) k^{4}+O\left(k^{6}\right),
\end{aligned}
$$

and higher order terms in (4.53b), with the exception of the $k^{6}$ term in $X_{2}^{(1)}(k)$, are not amenable to calculation using our procedure of a straightforward expansion in $k$ of the integrand. Previous sum-rule arguments [14] gave correctly the leading $k^{4}$ dependence of the background contribution but were too crude to reveal the leading $k^{4} \ln (1 / k)$ dependence of $X_{-1}^{(\mathbf{1})}(k)$. The behavior of $X_{m}^{(1)}(k)$ for $m \geqslant 3$ will be discussed below.

We now systematically check that the important sum rules involving $g \mathscr{S}(k, \omega)$ are satisfied to first order in $g$. We first consider the compressibility sum rule

$$
\lim _{k \rightarrow 0} g \chi(k)=\lim _{k \rightarrow 0}\left(-2 g \mathscr{S}_{-1}(k)\right)=-1 / c^{2},
$$

where $c$ is the macroscopic sound speed. From $(4.5 \mathrm{lb})$ and $(4.54)$, we find the nonanalytic expression

$$
\begin{aligned}
g \chi(k)= & \left(-1+g 2 / \pi^{2}\right)+\left(\frac{1}{4}-g 161 / 144 \pi^{2}\right) k^{2} \\
& -g\left(43 / 3840 \pi^{2}\right) k^{4} \ln (1 / k)+O\left(k^{4}\right) .
\end{aligned}
$$

In the $k \rightarrow 0$ limit, $g \chi(k) \rightarrow-1+g 2 / \pi^{2}=-1 / c_{0}{ }^{2}$ and (4.54) is satisfied if $c^{2}=c_{0}{ }^{2}$. The macroscopic sound speed can be easily calculated from the ground state energy $[4,5]$ to give

$$
1 / c^{2}=1-g 2 / \pi^{2}+O\left(g^{2}\right)=1 / c_{0}^{2} .
$$

Thus we see to order $g$ the coincidence of the macroscopic sound speed with the zero sound speed as well as the satisfaction of the compressibility sum rule. The other important sum rule is the $f$ sum rule, (4.31). From (4.51b) we can verify 
explicitly to first order in $g$ that the $f$ sum rule is satisfied up through terms proportional to $g k^{6} \ln k$. However by examining the asymptotic behavior of $\mathscr{F}^{(1)}(\mathbf{k}, \omega)$, we can now easily show that the $f$ sum rule is satisfied to $O(g)$ for all values of $k$. Since from (4.10) $Q^{+} \sim \omega^{-2}$ and $Q^{-} \sim \omega^{-1}$ as $\omega \gg p^{2} \gg 1$, we see from (3.32) and (4.4)-(4.9) that as $\omega \rightarrow \infty$ the leading dependence of $\mathscr{N}^{z z(1)}(\mathbf{k}, \omega)$ appears to be proportional to $\omega^{2}$. But the coefficient has a linear large- $p$ divergence from terms like $v^{(\mathbf{1})}$ [see (4.22) and (4.23)], which arises from the point nature of our model interaction. From the inequality $\omega \gg p^{2}$, we can cut off the integration over $p$ at $\sim \omega^{1 / 2}$. Hence we find that the asymptotic behavior is given by $\mathscr{N}^{z z(1)}(\mathbf{k}, \omega) \sim \omega^{5 / 2}$ and from (3.31) $\mathscr{F}^{(1)}(\mathbf{k}, \omega) \sim \omega^{-7 / 2}$. Combining this result for $\mathscr{F}^{(1)}$ with that for $\mathscr{F}^{(0)}$ from (3.11), we obtain as $\omega \rightarrow \infty$

$$
g \mathscr{F}(\mathbf{k}, \omega) \rightarrow \mathbf{k}^{2} \omega^{-2}+O\left(g \omega^{-7 / 2}\right) .
$$

The asymptotic form of $\mathscr{F}(\mathbf{k}, \omega)$ can be written (see Appendix D)

$$
g \mathscr{F}(\mathbf{k}, \omega) \rightarrow 2 \omega^{-2} g \mathscr{S}_{1}(k)+O\left(g \omega^{-7 / 2}\right) .
$$

Comparing (4.57a) with (4.57b), we see that

$$
g \mathscr{T}_{1}(k)=\frac{1}{2} k^{2}+O\left(g^{2}\right),
$$

and the $f$ sum rule is explicitly verified to first order in $g$. The sum rule

$$
\int_{0}^{\infty} d \omega \omega^{-1} g \mathscr{S}^{z z}(\mathbf{k}, \omega)=\frac{1}{2}
$$

where $\mathscr{S}^{z z}(\mathbf{k}, \omega)=-\pi^{-1} \operatorname{Im} \mathscr{F}^{z z}(\mathbf{k}, \omega)$, is equivalent to the $f$ sum rule because of the identity (2.31). Other first $\omega$-moment sum rules are discussed in Appendix D. Hence we have seen explicitly that to first order in $g$ it is possible to obtain an elementary excitation spectrum without a gap (Section 4B) and to verify the important sum rules related to number conservation.

We now consider $\mathscr{S}_{m}(k)$ for $m=0$ and $m=2$. From (4.51b) we see that the static structure function $\mathscr{S}_{0}(k)$ has the form

$$
\begin{aligned}
g \mathscr{S}_{0}(k) & =k\left[s_{0}+s_{2} k^{2}+s_{3} k^{3}+O\left(k^{4}\right)\right], \\
s_{0} & =\frac{1}{2}-g / 2 \pi^{2} \\
s_{2} & =-1 / 16+g\left(103 / 480 \pi^{2}\right), \\
s_{3} & =g(7 / 10240 \pi) .
\end{aligned}
$$

The numerical coefficients given in Ref. [9] are correct, but the cancellation of the $k^{5} \ln (1 / k)$ terms contributing to $\mathscr{S}_{0}(k)$, which is made explicit in $(4.51 \mathrm{~b})$, was overlooked. The one-phonon and backflow terms in $\mathscr{S}(k, \omega)$ both make contri- 
butions to $\mathscr{S}_{F}^{(1)}(k)$ proportional to $k^{5} \ln k$ but the contributions cancel exactly. Thus the contribution of $Z_{F}^{(1)}(k)+Y^{(1)}(k)$ can be represented by a power series through order $k^{5},(4.52)$, but we have not calculated the numerical coefficient of the $k^{\bar{b}}$ term nor the form of higher terms in $k$. Also $X_{0}^{(1)}(k)$ has not been investigated beyond order $k^{4}$. Thus $\mathscr{S}_{0}(k)$ has the form displayed in (4.59). We see from (4.59) that $\mathscr{S}_{0}(k)$ has both even and odd powers of $k$ and displays no logarithm through the $k^{5}$ term. The background term $X_{0}^{(1)}(k),(4.53 \mathrm{~b})$, gives rise to the $s_{3} k^{4}$ term in (4.59); and the backflow term $Y^{(1)},(4.38)$, contributes to the $s_{2} k^{3}$ term in (4.59). We thus conclude that because of backflow the Feynman relation

$$
g \cdot \mathscr{S}_{0}(k)=k^{2} / 2 \omega_{k}
$$

breaks down at the $s_{2} k^{3}$ term. From $(4.51 b)$ we see that $\mathscr{S}_{2}(k)$ has the form

$$
\begin{aligned}
g \mathscr{S}_{2}(k)= & \frac{1}{2}\left(1+\frac{g}{\pi^{2}}\right) k^{3}+g\left(\frac{7}{480 \pi}\right) k^{4} \\
& +\left(\frac{1}{16}+g \frac{143}{1440 \pi^{2}}\right) k^{5}+O\left(g k^{6}\right)+O\left(g^{2} k^{3}\right)
\end{aligned}
$$

The background contribution $X_{2}^{(1)}(k)$ has in addition to the leading $k^{4}$ contribution (4.53b), a $k^{6}$ contribution, which has not been calculated because the calculation is a tedious one.

The large $\omega$ behavior of $X^{(\mathbf{1})}(\mathbf{k}, \omega)$ given in (4.48) implies that the coefficient of the leading small $k$ term of $X_{m}^{(1)}(k)$ and hence $\mathscr{F}_{m}^{(1)}(k)$ is divergent for $m \geqslant 3$. This divergence leads to the conclusion that it is not possible to calculate $\mathscr{S}_{m}(k)$ for $m \geqslant 3$ for a model in which the interaction is represented solely by a scattering length and that an additional parameter is necessary to ensure that $\mathscr{T}_{m}(k)$ for $m \geqslant 3$ is finite. It is clear from (4.48) that a large $w$ (short-time) cut-off is needed. Since the present calculation is in the collisionless regime and does not describe real collisions (see Section 5C), the relevant short-time cut-off is the time of collision $\tau$, not the time between collisions. It is then reasonable to assume that (4.48) is valid for $\omega<1 / \tau$. If the dilute Bose gas has a hard-core interaction, $\tau$ vanishes and the divergence of $\mathscr{S}_{3}(k)$ found to first order in $g$ is consistent with the general result reported by Kleban [15]. However if the dilute Bose gas has a smooth potential, $\tau$ does not vanish and $\mathscr{S}_{3}(k)$ does not diverge. We note that for all the physical quantities discussed thus far with the exception of $\mathscr{S}_{m}^{(1)}(k)$ for $m \geqslant 3$, the effects of the interaction can be described by one parameter and our expansion is valid through first order in $g$. The functions $\mathscr{T}_{m}(k)$ for $m \geqslant 3$ give more weight to the high-energy or short-time behavior of the system and make it necessary to introduce additional parameters to describe the short-time effects of the interaction. A similar problem with the first $\omega$-moment of the singular dynamic structure function is discussed in Appendix D. 


\section{E. The One-Particle Spectral Function}

The one-particle spectral function $\mathscr{L}(\mathbf{k}, \omega)$ is defined for all $\omega$ as

$$
\mathscr{H}(\mathbf{k}, \omega)=-\frac{1}{\pi} \operatorname{Im} \mathscr{G}_{++}(\mathbf{k}, \omega)
$$

Although $\mathscr{A}(\mathbf{k}, \omega)$ has not been measured directly, an explicit calculation of $\mathscr{A}(\mathbf{k}, \omega)$ will show clearly the close relationship between $\mathscr{A}(\mathbf{k}, \omega)$ and $\mathscr{S}(\mathbf{k}, \omega)$ in a Bose system. Furthermore we can explicitly demonstrate that the present calculation satisfies various sum rules for $\mathscr{L}(\mathbf{k}, \omega)$.

We see from (3.13) and (3.14) and (4.62) that the zeroth order $\mathscr{A}^{(0)}(\mathbf{k}, \omega)$ can be written as

$$
\begin{aligned}
k^{2} \circlearrowleft \mathcal{X}^{(0)}(\mathbf{k}, \omega)= & Z^{(0)}(k)\left[\left(\omega_{k}^{(0)}+1+\epsilon_{k}\right) \delta\left(\omega-\omega_{k}^{(0)}\right)\right. \\
& \left.+\left(\omega_{k}^{(0)}-1-\epsilon_{k}\right) \delta\left(\omega+\omega_{k}^{(0)}\right)\right] \\
= & \left(\omega+1+\epsilon_{k}\right)\left[\mathscr{S}^{(0)}(\mathbf{k}, \omega)-\mathscr{S}^{(0)}(\mathbf{k},-\omega)\right],
\end{aligned}
$$

where $\mathscr{S}^{(0)}(\mathbf{k}, \omega)$ is given by $(4.28)$.

The first-order term $\mathscr{A}^{(1)}(\mathbf{k}, \omega)$ is obtained from the first-order result (3.41)-(3.44) for $k^{2} \mathscr{G}_{++}^{(1)}$. If we take the imaginary part of $\mathscr{G}_{++}^{(\mathbf{1})}$, we find

$$
k^{2} \mathscr{A}^{(\mathbf{1})}(\mathbf{k}, \omega)=\left(\omega+1+\epsilon_{k}\right)\left[\mathscr{S}^{(\mathbf{1})}(\mathbf{k}, \omega)-\mathscr{S}^{(\mathbf{1})}(\mathbf{k},-\omega)\right]+\mathscr{T}^{(1)}(\mathbf{k}, \omega)
$$

where $\mathscr{S}^{(1)}(\mathbf{k}, \omega)$ is given by $(4.30)$. The function $\mathscr{T}^{(1)}(\mathbf{k}, \omega)$ is defined by

$$
\begin{aligned}
\mathscr{T}^{(1)}(\mathbf{k}, \omega) & =-\frac{1}{\pi} \operatorname{Im} \mathscr{H}^{(1)}(\mathbf{k}, \omega) \\
& =-\frac{1}{\pi} \operatorname{Im}\left[\left(\omega^{2}-\omega_{k}^{2(0)}\right)^{-1} \mathscr{I}^{z z(1)}(\mathbf{k}, \omega)\right],
\end{aligned}
$$

where $\mathscr{H}^{(\mathbf{1})}$ and $\mathscr{I}^{2 z(\mathbf{1})}$ are given by (3.42)-(3.45).

Equation (4.64) emphasizes the close relationship between $\mathscr{A}(\mathbf{k}, \omega)$ and $\mathscr{S}(\mathbf{k}, \omega)$. First $\mathscr{G}_{++}$and $\mathscr{F}$ share the same singularities and hence both functions have a gapless spectrum. Furthermore from the first term in (4.64), we see that the relative weights for the analogous singularities in $\mathscr{G}_{++}$and $\mathscr{F}$ are related to each other, including that for the incoherent background. Thus the first term in (4.64) is an explicit representation of the sharing of singularities between $\mathscr{G}_{++}$and $\mathscr{F}$. The last term in (4.64), $\mathscr{T}(\mathbf{k}, \omega)$, represents contributions to $\mathscr{A}(\mathbf{k}, \omega)$ that do not come directly from $\mathscr{S}(\mathbf{k}, \omega)$ and may be called the intrinsic one-particle spectral function. 
Since $\operatorname{Im}\left(\omega^{2}-\omega_{k}^{2(0)}\right)^{-1}-(\pi / 2 \omega)\left[\delta\left(\omega-\omega_{k}^{(0)}\right)+\delta\left(\omega+\omega_{k}^{(0)}\right)\right]$, we can write the intrinsic spectral function in the form

$$
\mathscr{\gamma}^{(\mathbf{1})}(\mathbf{k}, \omega)=U^{(1)}(k) \delta\left(\omega-\omega_{k}^{(0)}\right)+V^{(1)}(k) \delta\left(\omega+\omega_{k}^{(0)}\right)+W^{(1)}(k, \omega),
$$

where

$$
\begin{aligned}
U^{(1)}(k) & =\left[\frac{1}{2 \omega} \mathscr{I}^{z z(1)}(\mathbf{k}, \omega)\right]_{\omega=-\omega_{k}^{(0)}}, \\
V^{(1)}(k) & =\left[\frac{1}{2 \omega} \mathscr{I}^{z z(1)}(\mathbf{k}, \omega)\right]_{\omega=-\omega_{k}^{(0)}}, \\
W^{(\mathbf{1})}(\mathbf{k}, \omega) & =\frac{1}{\omega^{2}-\omega_{k}^{2(0)}}\left[-\frac{1}{\pi} \operatorname{Im} \mathscr{I}^{z z(1)}(\mathbf{k}, \omega)\right] .
\end{aligned}
$$

If we use (3.43)-(3.45) we can numerically evaluate $U^{(1)}(k)$ and $V^{(1)}(k)$ following the same procedure as before and obtain

$$
\begin{aligned}
& \frac{1}{2}\left[U^{(1)}(k)+V^{(1)}(k)\right]=-\frac{1}{4 \pi^{2}} k^{2}-\frac{1}{32 \pi^{2}} k^{4} \ln (1 / k)+O\left(k^{1}\right), \\
& \frac{1}{2}\left[U^{(1)}(k)-V^{(1)}(k)\right]-\frac{5}{6 \pi^{2}} k-\frac{71}{180 \pi^{2}} k^{3}-\frac{9}{320 \pi^{2}} k^{5} \ln (1 / k)+O\left(k^{5}\right) .
\end{aligned}
$$

It might be thought that the coherent one-phonon part of $\mathscr{S}^{(1)}(\mathbf{k}, \omega)$, which is included in $\mathscr{A}^{(\mathbf{1})}(\mathbf{k}, \omega)$ (see (4.64)), can be identified as the coherent one-phonon part of $\mathscr{A}^{(1)}$. However we see from (4.66) and (4.68) that the intrinsic spectral function $\mathscr{T}^{(1)}(\mathbf{k}, \omega)$ contains a coherent part which has the same leading $k$ dependence as $Z^{(1)}(k)$, i.e., both $Z^{(1)}(k)$ and $U^{(1)}(k)$ are proportional to $k$ for small $k$. Thus, the proposed identification of the one-phonon part of $\mathscr{A}^{(1)}$ is not correct.

We now examine the structure of the background term $W^{(1)}(\mathbf{k}, \omega)$. Using the arguments leading to the threshold for $X^{(1)}(\mathbf{k}, \omega)$, we find that both delta functions in (4.41) contribute to $W^{(1)}(\mathbf{k}, \omega)$ since $W^{(1)}$ is nonzero for both positive and negative values of $\omega$. Thus we find that for fixed $k, W^{(1)}(\mathbf{k}, \omega)$ vanishes in the range $2 \omega_{k / 2}^{(0)} \geqslant \omega \geqslant-2 \omega_{k / 2}^{(0)}$; in other words the threshold is given by

$$
\omega_{t h r}^{(0)}- \pm 2 \omega_{k: 2}^{(0)} \text {. }
$$

Similarly it is easy to show as in (4.43) that the branch points at the threshold (4.69) for $W^{(\mathbf{1})}(\mathbf{k}, \omega)$ and hence $\mathscr{\mathscr { A }}^{(\mathbf{1})}(\mathbf{k}, \omega)$ are square-root singularities. We can also 
determine the behavior of $W^{(1)}(\mathbf{k}, \omega)$ in the limit of large $\omega$ by first dividing $W^{(1)}$ into even and odd parts with respect to $\omega$

$$
\begin{aligned}
W^{(1)}(\mathbf{k}, \omega) & =W_{+}^{(1)}(\mathbf{k}, \omega)+W_{-}^{(1)}(\mathbf{k}, \omega), \\
W_{ \pm}^{(1)}(\mathbf{k}, \omega) & =-\left(\omega^{2}-\omega_{k}^{2(0)}\right)^{-1} \pi^{-1} \operatorname{Im} \mathscr{I}_{\mp}^{z z(1)}(\mathbf{k}, \omega) \\
& = \pm W_{ \pm}^{(1)}(\mathbf{k},-\omega) .
\end{aligned}
$$

Then using the same arguments leading to (4.48), we find for large positive $\omega \gg k^{2} \gg 1$

$$
\begin{aligned}
& W_{+}^{(1)}(\mathbf{k}, \omega)=\left(1 / 4 \pi^{2}\right)\left(k^{2} / \omega^{3 / 2}\right)\left[1+61 k^{2} / 60 \omega+O\left(k^{4} / \omega^{2}\right)\right] \\
& W_{-}^{(1)}(\mathbf{k}, \omega)=\left(1 / 4 \pi^{2}\right)\left(k^{2} / \omega^{3 / 2}\right)\left[1+5 k^{2} / 4 \omega+O\left(k^{4} / \omega^{2}\right)\right] .
\end{aligned}
$$

Note that for negative $\omega$ the leading terms in $W_{+}^{(1)}$ and $W_{-}^{(1)}$ exactly cancel in $W^{(1)}$, and we have for $|\omega| \gg k^{2} \gg 1$

$$
W^{(1)}(\mathbf{k}, \omega)= \begin{cases}\left(1 / 2 \pi^{2}\right) k^{2} / \omega^{3 / 2}, & \omega>0 \\ -\left(7 / 120 \pi^{2}\right) k^{4} /|\omega|^{5 / 2}, & \omega<0 .\end{cases}
$$

From (4.48), (4.64), (4.66), and (4.72) we find that the spectral function $\mathscr{A}(\mathbf{k}, \omega)$ has the following behavior for $|\omega| \geqslant k^{2} \geqslant 1$

$$
\mathscr{f}^{(1)}(\mathbf{k}, \omega)= \begin{cases}\left(1 / 2 \pi^{2}\right) \omega^{-3 / 2}, & \omega>0 \\ -\left(7 / 60 \pi^{2}\right) k^{2}|\omega|^{-5 / 2}, & \omega<0 .\end{cases}
$$

Note that the sign of $\mathscr{A}^{(1)}(\mathbf{k}, \omega)$ in (4.73) is consistent with the general property of Bose systems that $\mathscr{A}(\mathbf{k}, \omega)$ is positive for $\omega>0$ and negative for $\omega<0$.

We now explicitly verify that the sum rules for $\mathscr{A}(\mathbf{k}, \omega)$ are satisfied to first order in $g$. We first consider the sum rule

$$
\int_{-\infty}^{\infty} d \omega \mathscr{L}(\mathbf{k}, \omega)=1
$$

It is easy to see that $\mathscr{A}^{(0)}(\mathbf{k}, \omega),(4.63)$, already exhausts (4.74) so that it is necessary to show that $\int_{-\infty}^{\infty} d \omega \mathscr{A}^{(\mathbf{1})}(\mathbf{k}, \omega)=0$. The first term in (4.64) does not contribute to the integral since (as was shown in Section $4 \mathrm{C}$ ) $\int_{0}^{\infty} d \omega \omega \mathscr{S}^{(1)}(\mathbf{k}, \omega)=0$ and $\mathscr{S}(\mathbf{k}, \omega)$ is an odd function of $\omega$. Thus we are left with the task of showing that

$$
\int_{-\infty}^{\infty} d \omega \mathscr{T}^{(1)}(\mathbf{k}, \omega) d \omega=0
$$

or

$$
g\left[U^{(1)}(k)+V^{(1)}(k)\right]+\int_{-\infty}^{\infty} g W_{+}^{(1)}(\mathbf{k}, \omega) d \omega-0
$$


If we use (4.67) and the same procedure used earlier, we can calculate that

$$
\int_{-\infty}^{\infty} W_{+}^{(1)}(\mathbf{k}, \omega) d \omega=k^{2} / 2 \pi^{2}+\left(1 / 16 \pi^{2}\right) k^{4} \ln (1 / k)+O\left(k^{4}\right)
$$

Hence comparing (4.68) and (4.76), we see that (4.75) and hence the sum rule (4.74) is satisfied to $O\left(g k^{4}\right)$.

We next consider an inverse-moment sum rule for $\mathscr{A}(\mathbf{k}, \omega)$. We define

$$
\chi_{A}(\mathbf{k})=k^{2} \int_{-\infty}^{\infty} d \omega \omega^{-1} \mathscr{M}(\mathbf{k}, \omega)
$$

then

$$
\lim _{k \rightarrow 0} \chi_{A}(k)=n_{0} / \rho_{s}=g n_{0},
$$

where $\rho_{s}$ is the superfluid density. The last equality in (4.78) holds only for $T=0$ where $\rho_{s}=n=1 / g$. We write $\chi_{A}(k)=\chi_{A}^{(0)}(k)+g \chi_{A}^{(1)}(k)+\cdots$. It is easy to show that $\chi_{A}^{(0)}(k)=k^{2}\left(1+\epsilon_{k}\right) / \omega_{k}^{2(0)}=1+k^{2} / 4+\cdots$ and $\chi_{A}^{(1)}(k \rightarrow 0)=-1 / 3 \pi^{2}$. Thus we find

$$
\lim _{k \rightarrow 0} \chi_{A}(k)=1-g / 3 \pi^{2}+O\left(g^{2}\right),
$$

which agrees with the first-order calculation of $g n_{0}$ (see (2.5) and (4.12)), and the sum rule (4.78) is satisfied. The sum rule involving the first $\omega$-moment of $\mathscr{A}(\mathbf{k}, \omega)$ is discussed in Appendix D.

The momentum distribution $n_{k}{ }^{\prime}$ can be calculated from $\mathscr{A}(\mathbf{k}, \omega)$ via the relation

$$
n_{k^{\prime}}=-\int_{-\infty}^{0} d \omega \cdot \mathscr{L}(\mathbf{k}, \omega)
$$

From (4.63) and (4.64), we can write $\mathscr{A}(\mathbf{k}, \omega)$ in the form

$$
\begin{aligned}
k^{2} \mathscr{A}(\mathbf{k}, \omega)= & \left(\omega+1+\epsilon_{k}\right)[g \mathscr{S}(\mathbf{k}, \omega)-g \mathscr{S}(\mathbf{k},-\omega)] \\
& +g^{\mathscr{T}}{ }^{(1)}(\mathbf{k}, \omega)+O\left(g^{2}\right),
\end{aligned}
$$

where $g \mathscr{P}(\mathbf{k}, \omega)$ is given by (4.50) and $\mathscr{T}^{(1)}(\mathbf{k}, \omega)$ by (4.66). Substituting (4.81) into (4.80) and using the sum rules (4.33) and (4.75), we can write $n_{k}{ }^{\prime}$ in the form

$$
\begin{aligned}
n_{k^{\prime}}^{\prime}= & \left(k^{-2}+\frac{1}{2}\right) g \cdot \mathscr{S}_{0}(k)-\frac{1}{2}+g k^{-2} \frac{1}{2}\left[U^{(1)}(k)-V^{(1)}(k)\right] \\
& +g k^{-2} \int_{0}^{\infty} d \omega W_{-.}^{(1)}(\mathbf{k}, \omega)+O\left(g^{2}\right)
\end{aligned}
$$


where $g \cdot \mathscr{P}_{0}(k)$ is given by $(4.59), \frac{1}{2}\left[U^{(1)}(k)-V^{(1)}(k)\right]$ by $(4.68 \mathrm{~b})$, and

$$
\int_{0}^{\infty} d \omega W_{-}^{(1)}(\mathbf{k}, \omega)=\frac{3}{32 \pi} k^{2}+\cdots
$$

We obtained (4.83) by using our $k$-expansion procedure; however, we have not been able to calculate the higher-order $k$-dependence. From (4.59), (4.68b), (4.82) and (4.83), we find $n_{k}{ }^{\prime}$ in the long-wavelength limit

$$
\begin{aligned}
n_{k}{ }^{\prime} & =k^{-1}\left[r_{0}+r_{1} k+r_{2} k^{2}+\cdots\right], \\
r_{0} & =\frac{1}{2}+g / 3 \pi^{2}, \\
r_{1} & =-\frac{1}{2}+g(3 / 32 \pi), \\
r_{2} & =3 / 16-g\left(619 / 1440 \pi^{2}\right) .
\end{aligned}
$$

Note that the intrinsic one-particle background $W_{-}(\mathbf{k}, \omega)$ contributes to the $r_{\mathbf{1}}$ term, whereas the density background $X(\mathbf{k}, \omega)$ contributes to the $r_{2} k$ term. However, because of the uncertainty in the higher-order $k$-dependences in (4.83), the coefficient $r_{2}$ may have an additional contribution to $O(g)$.

Finally it is instructive to compare our perturbation results for $\mathscr{A}(\mathbf{k}, \omega)$ to the exact long-wavelength result of Gavoret and Nozieres [1]

$$
\lim _{k \rightarrow 0} k^{2} \mathscr{A}(\mathbf{k}, \omega)=\frac{1}{2} g n_{0} c k[\delta(\omega-c k)-\delta(\omega+c k)]
$$

where $c$ is the macroscopic sound speed. We have from (4.63)

$$
\lim k^{2} \mathscr{A}^{(0)}(\mathbf{k}, \omega)=\frac{1}{2} k[\delta(\omega-k)-\delta(\omega+k)]
$$

and from (4.34), (4.64), (4.66), and (4.68)

$$
\begin{aligned}
\lim _{k \rightarrow 0} k^{2} g \cdot \mathscr{L}^{(1)}(\mathbf{k},(\omega)= & -\frac{1}{2} \pi^{-2} g k\left[\delta(\omega-k)+k \delta^{\prime}(\omega-k)\right. \\
& \left.-\delta(\omega+k)+k \delta^{\prime}(\omega+k)\right] \\
& +\frac{5}{6} \pi^{-2} g k[\delta(\omega-k)-\delta(\omega+k)] .
\end{aligned}
$$

We can combine (4.86) and (4.87) and write $\mathscr{A}(\mathbf{k}, \omega)$ in the form

$$
\begin{aligned}
\lim _{k \rightarrow 0} k^{2} \mathscr{X}(\mathbf{k}, \omega)= & \frac{1}{2} k\left(1+g 2 / 3 \pi^{2}\right)\left[\delta\left(\omega-k\left(1+g / \pi^{2}\right)\right)\right. \\
& \left.-\delta\left(\omega+k\left(1+g / \pi^{2}\right)\right)\right],
\end{aligned}
$$

which agrees with the expansion of (4.85) to first order in $g$ using $g n_{0}=1-g / 3 \pi^{2}$, (4.12), and $c=1+g / \pi^{2}(4.56)$. 


\section{Discussion}

The many unexpected results obtained in Section 4 for the Bose gas to first order in $g$ naturally lead us to consider whether some of the qualitative features found for various physical quantities in the model calculation might also be found in the analogous quantities in superfluid helium. Of course the numerical coefficients obtained in the model calculation will not be useful in fitting data of helium. Furthermore it should be cautioned that the form of physical quantities obtained in the limit of $k \rightarrow 0$ might not be relevant to fitting data taken at finite values of $k$.

In Section $5 \mathrm{~A}$ we suggest that logarithmic terms can be expected to be present in various physical quantities such as the excitation spectrum $\omega_{k}$. In Section $5 B$ we consider a simple qualitative analysis of the form of $\mathscr{F}(\mathbf{k}, \omega)$ and give simple sum rule arguments that indicate that the Feynman one-phonon relation between $\mathscr{S}_{0}(k)$ and $\omega_{k}$ breaks down at order $k^{3}$. Finally in Section 5C we summarize some of the physical features of the generalized dielectric formulation and suggest future work.

\section{A. Logarithmic Terms}

Mathematically the reason that the present first-order calculation is of interest is that in this approximation the response functions have analytic properties, e.g. branch cuts, which are expected to be present in a fully renormalized theory. The first-order calculation uncovered logarithmic terms $\left(\propto k^{n} \ln (1 / k)\right)$ in many functions of $k$, in particular $\omega_{k}$. We now present an argument that suggests that $\omega_{k}$ is not a analytic function of $k$ for a Bose liquid. Define $F_{\text {pair }}^{z z}$ as that part of $F^{z z}$ that has an isolated pair of single-particle lines. We can write

$$
F_{\text {pair }}^{z z} \propto i \int \frac{d^{4} p}{(2 \pi)^{4}}((\mathbf{p}+\mathbf{k} / 2) \cdot \hat{\mathbf{k}})^{2} \Gamma^{2} \mathscr{G}(\rho) \mathscr{G}(p+k),
$$

where $p=(\mathbf{p}, \epsilon), k=(\mathbf{k}, \omega)$, the first factor $(\mathbf{p}+\mathbf{k} / 2) \cdot \hat{\mathbf{k}}$ arises from the longitudinal current, and the vertex function $\Gamma$ is determined by the Ward identity $\Gamma=\partial \mathscr{G}-1 / \partial \omega$. Gavoret and Nozieres [1] have shown to all orders in perturbation theory that in the long wavelength limit the (causal) Green's function has the form $\mathscr{G}^{-1}=\left(n / n_{0} m c^{2}\right)\left(\omega^{2}-c^{2} k^{2}+i 0+\right)$, where $c$ is the macroscopic sound speed; and thus $\Gamma \propto \omega$ in the long-wavelength limit. If we evaluate (5.1) using the longwavelength form of $\mathscr{G}$ and $\Gamma$, we find that for small $k$ and $\omega$ the real part of $F_{\text {pair }}^{z z}$ contains a singular term of the form

$$
\operatorname{Re} F_{\text {pair }}^{z z} \propto \omega^{4} \ln \left|\omega^{2}-c^{2} k^{2}\right| .
$$

The logarithmic singularity (5.2) is similar in form to that found by Gavoret and Nozieres [1], who assumed that this type of singularity is unphysical but were 
only able to show that it disappears from physical quantities to leading order in $k$. The logarithmic term $k^{5} \ln (1 / k)$ in $\omega_{k}$ can be obtained from (4.15) and (5.2) if we make the simple assumption that $\omega_{k}$ must deviate from a linear dispersion relation. Note that the appearance of a logarithmic term in $\omega_{k}$ is independent of the nature of the deviation of $\omega_{k}$ from a linear spectrum. In contrast, the form of $\operatorname{Im} \omega_{k}$ depends on the nature of the dispersion of $\omega_{k}$ as shown in Appendix C.

\section{B. Form of $\mathscr{S}(\mathbf{k}, \omega)$ and the Breakdown of the Fevnman Relation}

In the present perturbative calculation, we have written $\mathscr{P}(\mathbf{k}, \omega)$ in the form given by (4.50), where the first-order corrections to the Feynman relation for the static structure function,

$$
g \mathscr{S}_{0}(k)=Z_{F}(k) \equiv k^{2} / 2 m \omega_{k},
$$

are isolated into two terms, the backflow $Y^{(\mathbf{1})}(k)$ and the background contribution $X^{(1)}(\mathbf{k}, \omega)$. We now explore the possibility that a similar form can be written for superfluid helium by the use of simple sum rule arguments.

Recall that $\mathscr{S}(\mathbf{k}, \omega)$ defined by (4.26) can also be given by

$$
\mathscr{T}(\mathbf{k}, \omega)=\sum_{v}\left|\left(\rho_{k}^{+}\right)_{v \mathbf{0}}\right|^{2} \delta\left(\omega-\omega_{v 0}\right),
$$

where $\left(\rho_{k}{ }^{+}\right)_{\nu 0}$ and $\omega_{\nu 0}$ are the exact matrix elements and excitation frequencies corresponding to the excited state $|\nu\rangle$ that is composed of a superposition of $\nu$ phonons. The $f$ sum rule (4.31) can be written

$$
\sum_{v}\left|\left(\rho_{k^{+}}^{+}\right)_{v \mathbf{0}}\right|^{2} \omega_{v \mathbf{0}}=N k^{2} / 2 m
$$

where $N$ is the number of Bosons. Since the one-phonon state exhausts the $f$ sum rule in the small- $k$ limit, the excited states $|\nu\rangle$ can be conveniently divided into the one-phonon state $|1\rangle$ and the multi-phonon state $|n\rangle$. Separating out the onephonon state in the $f$ sum rule (5.5), we find

$$
\left|\left(\rho_{k}{ }^{+}\right)_{10}\right|^{2}=N k^{2} / 2 m \omega_{k}-\omega_{k}^{-1} \sum_{n}\left|\left(\rho_{k}^{+}\right)_{n 0}\right|^{2} \omega_{n 9},
$$

where $\omega_{k} \equiv \omega_{10}$ is the one-phonon spectrum. Inserting (5.6) into (5.5), we find a natural three-fold division of $\mathscr{S}(\mathbf{k}, \omega)$

$$
\begin{aligned}
N^{-1} \mathscr{P}(\mathbf{k}, \omega) & =Z_{F}(k) \delta\left(\omega-\omega_{k}\right)+Y(k) \delta\left(\omega-\omega_{k}\right)+X(\mathbf{k}, \omega), \\
Z_{F}(k) & \equiv k^{2} / 2 m \omega_{k}, \\
Y(k) & \equiv-\omega_{k}^{-1} N^{-1} \sum_{n}\left|\left(\rho_{k}^{+}\right)_{n 0}\right|^{2} \omega_{n 0}, \\
X(\mathbf{k}, \omega) & \equiv N^{-1} \sum_{n}\left|\left(\rho_{k}^{+}\right)_{n \mathbf{0}}\right|^{2} \delta\left(\omega-\omega_{n 0}\right) .
\end{aligned}
$$


$Z_{F}(k)$ is evidently the one-phonon contribution. From $(5.8 \mathrm{~b})$ we see that the contribution $Y(k)$ arises from the virtual excitation of multiphonons, which is the usual microscopic picture of backflow [14]. In contrast the background term $X(\mathbf{k}, \omega)$ given by $(5.8 \mathrm{c})$ arises from the excitation of real multiphonons. In Eqs. (4.34), (4.39), and (4.40) and the discussion thereafter, we find exactly the same division and interpretation of $\mathscr{S}(\mathbf{k}, \omega)$ as in (5.7) and (5.8) but within perturbation theory. Hence (5.7) and (5.8) verify and extend to superfluid helium the present perturbation calculation of $\mathscr{S}(\mathbf{k}, \omega)$.

In general discussions of $\mathscr{P}(\mathbf{k},(t)$ in superfluid helium [14], $\mathscr{T}(\mathbf{k}, \omega)$ is usually divided into two terms

$$
N^{-1} \mathscr{S}(\mathbf{k}, \omega)-Z(k) \delta\left(\omega-\omega_{k}\right)+X(\mathbf{k}, \omega),
$$

where $Z(k)$ is interpreted as coming from "single-particle excitation" and $X(\mathbf{k}, \omega)$ coming from "multiparticle excitations." Contrary to this interpretation, we sce from (5.7) and (5.9) that $Z(k)$ has two contributions, $Z(k)=Z_{F}(k)+Y(k)$, and contains multiphonon contributions through the backflow $Y(k)$.

To be more quantitative, we consider the structure functions $\mathscr{S}_{m}(k)$ defined in (4.49), which can be written from (5.7) as

$$
\begin{aligned}
N^{-1} \mathscr{T}_{m}(k) & =Z_{F}(k) \omega_{k}^{m}+Y(k) \omega_{k}^{m}+X_{m}(k), \\
X_{m}(k) & \equiv N^{-1} \sum_{n} \mid\left(\rho_{k}^{+}\right)_{n 0} !^{2}\left(\omega_{n 0}\right)^{m} .
\end{aligned}
$$

Following Ref. [14], we use the continuity equation to write $\left(\rho_{k}{ }^{+}\right)_{n 0}=$ $\left(\mathbf{k} \cdot \mathbf{J}_{k}{ }^{+}\right)_{n \mathbf{0}} \omega_{n \mathbf{0}}^{-1}$. The $k \rightarrow 0$ limit of $\left(\mathbf{J}_{k}^{+}\right)_{n \mathbf{0}}$ is the matrix element of the total current, which vanishes by translational invariance. Expanding $\left(\mathbf{J}_{k}{ }^{+}\right)_{n 0}$ to first order in a Taylor series in $\mathbf{k}$, we find that $\left(\mathbf{J}_{k}{ }^{+}\right)_{n 0} \propto \mathbf{k}$. Since $\omega_{n 0}$ is finite in the $k \rightarrow 0$ limit, we find $\left(\rho_{k}{ }^{+}\right)_{n 0} \propto k^{2}$. From (5.8) we see that $Y(k) \propto k^{3}$ and from (5.11) that $X_{m}(k) \propto k^{4}$. Thus the Feynman relation (5.3) for superfluid heluim breaks down at the $k^{3}$ term because of backflow. Since the backflow $Y(k)<0$ and $\propto k^{3}$ and the background $X_{0}(k)>0$ and $\propto k^{4}$, we expect the static structure function $\mathscr{T}_{0}(k)$ in general to show at small $k$ an inflection point [14] due to the interplay between the negative backflow and positive background contributions.

From the above sum rule argument for $X_{m}(k) \propto k^{4}$ and Eq. (5.10) we see that the leading $k$-dependence of $\mathscr{S}_{m}(k)$ with $m \geqslant 3$ is determined either partially $(m=3)$ or completely $(m>3)$ by the multi-phonon background contribution $X_{m}(k)$. It is because of the considerable relative weight given to the high frequency multiphonons that even the long wavelength limit of $\mathscr{S}_{m \geqslant 3}(k)$ is not accounted for in the one-phonon Feynman picture, i.e., retaining in $\mathscr{T}(\mathbf{k}, \omega)$ only the $Z_{F}(k) \delta\left(\omega-\omega_{k}\right)$ term in (5.7). However these sum rule arguments are not capable of determining the proportionality constant in $\mathscr{S}_{m \geqslant 3}(k) \propto k^{4}$. In perturbation 
theory [cf. the discussion after (4.61)], we note that a finite result for $k^{-4} \mathscr{S}_{m \geqslant 3}(k)$ is obtained only after an ad hoc short-time cut off of the order of the time-ofcollision is introduced. Thus we conclude that for $\mathscr{S}_{m \geqslant 3}(k)$ the one-phonon Feynman picture breaks down completely and that a consistent treatment of multiphonon effects as well as short-time collisional effects may be necessary in order to analyze $\mathscr{S}_{m \geqslant 3}(k)$ properly.

\section{Concluding Remarks}

The present rigorous microscopic calculation of $\mathscr{S}(\mathbf{k}, \omega), \mathscr{A}(\mathbf{k}, \omega), \mathscr{S}_{m}(k), \omega_{k}$, and related functions was based upon a formalism which emphasizes some of the special properties of zero temperature Bose systems. The fact that the single particle response function $\mathscr{G}$, the density response function $\mathscr{F}$, and the current response function $\mathscr{F}^{z z}$ all share the same set of singularities allows us to express $\mathscr{G}$, $\mathscr{F}$, and $\mathscr{F}^{z z}$ in terms of the dielectric function $\epsilon(k, \omega)$. I he effects of backflow, important in higher order calculations, were automatically taken into account by including generalized Ward identities based upon the continuity equations into the structure of the formalism and were isolated by the use of the $f$ sum rule. In this manner the present formalism enables us to achieve perturbation approximations which obey local number conservation and related sum rules as well as give an excitation spectrum that is without a gap. The approach also allowed us to perform detailed calculations beyond the Bogoliubov approximation with relative ease and without spurious divergences.

As emphasized in Ref. [8], the present perturbation calculation of $\omega_{k}$ in the long-wavelength limit parallels the determination of zero sound in a Fermi gas and consequently the phonon in a Bose gas may be interpreted as zero or collisionless sound. The one-loop diagrams included in the present first-order calculation do not describe real collisions and hence all hydrodynamic modes have been ignored in our calculations of the various response functions. An important extension of the present work would be to include the hydrodynamic modes, e.g., second sound, into a calculation of $\mathscr{S}(\mathbf{k}, \omega)$ and $\mathscr{A}(\mathbf{k}, \omega)$ at nonzero temperatures.

The next order in the perturbation expansion, i.e., the two-loop diagrams, is also of interest since it would lead to a critical examination of the "four-phonon processes" and would indicate the role of close-range interactions. In this approximation many functions [5] cannot be evaluated using an interparticle interaction that is described solely by the scattering length. Because of the rather sizeable reduction in algebraic complexity in the present formalism, a calculation of the contribution of the two-loop diagrams to the spectrum $\omega_{k}$ appears to be feasible. 


\section{APPENDIX A-ONE-LOOP INTEGRALS}

The calculation of the integrals (4.1)-(4.3) corresponding to the one-loop diagrams in Fig. 6 follows straightforwardly from the rules of standard zerotemperature perturbation theory [13]. In the text we have not distinguished notationally between the response functions (2.11)-(2.17) which have simple analytical properties and the corresponding casual propagators for which a simple diagrammatic perturbation expansion exists, since at zero temperature the two functions are simply related by taking $\omega \rightarrow \omega=i 0+$ in the right places. For example in the integrals (4.1)-(4.3) $\mathscr{G}^{(0)} \mu \nu(p)$ is to be interpreted as the casual propagator. It is convenient to write $\mathscr{G}_{++}^{(0)}(p)$ and $\mathscr{G}_{+-}^{(0)}(p)$ in the form

$$
\begin{aligned}
\mathscr{G}_{++}^{(0)}(p) & =\frac{1+\eta_{p}}{\epsilon-\omega_{p}+i 0+}-\frac{\eta_{p}}{\epsilon+\omega_{p}-i 0+}, \\
\mathscr{G}_{+-}^{(0)}(p) & =-\bar{\eta}_{p}\left(\frac{1}{\epsilon-\omega_{p}+i 0+}-\frac{1}{\epsilon+\omega_{p}-i 0+}\right),
\end{aligned}
$$

where from (3.13) $1+\eta_{p}=u_{p}^{2}, \eta_{p}=v_{p}^{2}$, and $\bar{\eta}_{p}=u_{p} v_{p}$, and the superscript on $\omega_{\mathrm{p}}^{(0)}$ has been suppressed. Recall that for real $a$ and $b$

$$
i \int \frac{d \epsilon}{2 \pi} \frac{1}{\epsilon+\omega-a+i 0+} \frac{1}{\epsilon+b-i 0+}=\frac{-1}{\omega-a-b} .
$$

As an example, we perform the frequency integral in (4.2) using (A.1) and (A.2) and find straightforwardly

$$
\begin{aligned}
\Lambda_{+}^{z(1)}= & \int \frac{d^{3} p}{(2 \pi)^{3}}(\mathbf{p}+\mathbf{k} / 2) \cdot \hat{\mathbf{k}}\left\{\left[\left(1+\eta^{\prime}\right)(\eta-\bar{\eta})+\bar{\eta}^{\prime}(1+\eta-\bar{\eta})\right] Q_{\mathbf{1}}\right. \\
& \left.+\left[\eta^{\prime}(1+\eta-\bar{\eta})+\bar{\eta}^{\prime}(\eta-\bar{\eta})\right] Q_{2}\right\},
\end{aligned}
$$

where $Q_{1}=\left(\omega-\omega_{p}-\omega_{\mathbf{p}+\mathbf{k}}\right)^{-1}, \quad Q_{2}=-\left(\omega+\omega_{p}+\omega_{\mathbf{p}+\mathbf{k}}\right)^{-1}$, and $\eta=\eta_{p}$, $\eta^{\prime}=\eta_{\mathrm{p}+\mathbf{k}}$, etc. If we use the fact that $Q_{1}(-\omega)=Q_{2}(\omega), \Lambda_{-}^{z}(\mathbf{k}, \omega)=\Lambda_{+}^{z}(-\mathbf{k},-\omega)$, we can show that

$$
\begin{aligned}
\Lambda_{\mu}^{z(1)} \beta_{\mu}= & \int \frac{d^{2} p}{(2 \pi)^{3}}(\mathbf{p}+\mathbf{k} / 2) \cdot \hat{\mathbf{k}}\left[\left(1+\eta^{\prime}+\bar{\eta}^{\prime}\right)(\eta-\bar{\eta})\right. \\
& \left.+\left(\eta^{\prime}+\bar{\eta}^{\prime}\right)(1+\eta-\bar{\eta})\right] Q^{+}
\end{aligned}
$$

where $\beta_{\mu}=\operatorname{sgn}(\mu)$ and $Q^{+}=Q_{1}+Q_{2}$. The identifies $1+\eta-\bar{\eta}=(\lambda+1) / 2$, $\eta-\bar{\eta}=(\lambda-1) / 2,1+\eta+\bar{\eta}=\left(\lambda^{-1}+1\right) / 2, \eta+\bar{\eta}=\left(\lambda^{-1}-1\right) / 2$ can be used to rearrange the coefficient of $Q^{-}$in (A.4) into the more convenient form of (4.8). The frequency integrals in (4.1) and (4.3) can be evaluated in the same way to obtain the results of (4.4)-(4.9). 


\section{APPENDIX B. USEFUl EXPANSIONS FOR SMALl $k$}

We collect here a list of small- $k$ expansions for various quantities, which is useful in the evaluation of the long-wavelength limit of the one-loop integrals (4.4)-(4.9). In the following equations, $u$ is the cosine of the angle between $\mathbf{p}$ and $\mathbf{k}$, $D=1+p^{2} / 4$, and the superscript $(0)$ on $\omega_{\mathrm{p}}^{(0)}=p D^{1 / 2}$ is suppressed

$$
\begin{aligned}
\omega_{\mathbf{p}+\mathbf{k}} / \omega_{\mathbf{p}} & =1+\omega_{1} k+\omega_{2} k^{2}+\omega_{3} k^{3}+\omega_{4} k^{4}+\cdots, \\
\omega_{\mathbf{1}} & =\frac{u}{p}+\frac{p u}{4 D}, \\
\omega_{2} & =\frac{1-u^{2}}{2 p^{2}}+\frac{1+12 u^{2}}{8 D}-\frac{p^{2} u^{2}}{32 D^{2}}, \\
\omega_{3} & =\frac{u^{3}-u}{2 p^{3} D^{2}}+\frac{u^{3}}{8 p D^{3}}, \\
\omega_{4} & =\frac{-5 u^{4}+6 u^{2}-1}{8 p^{4} D^{2}}+\frac{-3 u^{4}+3 u^{2}}{16 p^{2} D^{3}}-\frac{5 u^{4}}{128 D^{4}} .
\end{aligned}
$$

Note that as $p \rightarrow 0 \quad \omega_{n} \propto p^{-n}$, but as $p \rightarrow \infty \quad \omega_{1} \rightarrow 2 u p^{-1}, \quad \omega_{2} \rightarrow p^{-2}$, $\omega_{3} \rightarrow 8\left(2 u^{3}-1\right) p^{-7}, \omega_{4} \rightarrow 2\left(-16 u^{4}+12 u^{2}-1\right) p^{-8}$. Hence in the large $p$ limit with $k / p$ fixed, (B.1) reduces to $\omega_{\mathrm{p}+\mathbf{k}} / \omega_{\mathrm{p}}=1+2 u(k / p)+(k / p)^{2}$ as expected.

$$
\begin{aligned}
{\left[\left(\omega_{\mathrm{p}}+\omega_{\mathrm{p}+\mathrm{k}}\right)^{2}-\omega_{\mathrm{k}}^{2}\right]^{-1} } & =\frac{1}{4 \omega_{p}^{2}}\left[1+a_{1} k+a_{2} k^{2}+a_{3} k^{3}+a_{4} k^{4}+\cdots\right], \\
a_{1} & =-\omega_{1}, \\
a_{2} & =\frac{5 u^{2}-1}{4 p^{2}}+\frac{2 u^{2}-3}{16 D}+\frac{5 u^{2} p^{2}}{64 D^{2}}, \\
a_{3} & =\frac{3 u-7 u^{3}}{4 p^{3}}+\frac{2 u-u^{3}}{2 p D}+\frac{u p\left(7-u^{2}\right)}{64 D^{2}}-\frac{7 p^{3} u^{3}}{256 D^{3}}, \\
a_{4} & =\frac{1}{8} p^{-4}\left(21 u^{4}-16 u^{2}+1\right)+O\left(p^{-2}\right) .
\end{aligned}
$$

Only the leading small-p dependence of $a_{4}$ in (B.2) is necessary for the present calculation

$$
\begin{aligned}
{\left[\left(\omega_{\mathbf{p}}+\omega_{\mathbf{p}+\mathbf{k}}\right)^{2}-\omega_{\mathbf{k}}^{2}\right]^{-2} } & =\frac{1}{16 \omega_{p}^{2}}\left[1+b_{1} k+b_{2} k^{2}+\cdots\right], \\
b_{1} & =-2 \omega_{1}, \\
b_{2} & =\frac{7 u^{2}}{2} p^{2} 1 \frac{6 u^{2}-3}{8 D}+\frac{7 p^{2} u^{2}}{32 D^{2}} .
\end{aligned}
$$


Note that this expansion obliterates the double pole (real part) on the left side of (B.3). Such double poles appear in one-loop integrals for $Y^{(1)}(k)$ and $X^{(1)}(k, \omega)$. If we evaluate these one-loop integrals without the use of $\{$ B.3), we find that the leading contributions from the neighborhood of the double pole cannot be neglected relative to the contributions from regions far removed from the double pole. The latter contributions, which are correctly given by (B.3), are obtained for $Y^{(1)}(k)$ and $X_{m}^{(1)}(k)$ in (4.38) and (4.53), respectively. Fortunately the leading double-pole contributions cancel when summed to give $\mathscr{S}^{(1)}(k, \omega)$ or $\mathscr{Y}_{m}^{(1)}(k)$. The use of (B.3) is justified to the extent that the contributions to $\mathscr{P}^{(1)}(k, \omega)$ from the double pole ignored in (B.3) are higher order than that obtained in (4.38) and (4.53).

$$
\begin{aligned}
\lambda_{\mathbf{p}+\mathbf{k}} / \lambda_{\mathbf{p}} & =1+\lambda_{1} k+\lambda_{\mathfrak{n}} k^{2}+\lambda_{3} k^{3}+\lambda_{1} k^{4}+\cdots \\
\lambda_{1} & =\frac{u}{p \bar{D}}, \\
\lambda_{2} & =\frac{1-u^{2}}{2 p^{2} D}-\frac{3 u^{2}}{8 D^{2}}, \\
\lambda_{3} & =\frac{u^{3}-u}{2 p^{3} D}+\frac{2 u^{3}-3 u}{8 p D^{2}}+\frac{5 p u^{3}}{32 D^{3}}, \\
\lambda_{4} & =\frac{-5 u^{1}+6 u^{2}-1}{8 p^{4} D}+\frac{-9 u^{4}+12 u^{2}-3}{32 p^{2} D^{2}}-\frac{15 u^{4}-30 u^{2}}{128 D^{3}}-\frac{35 p^{2} u^{4}}{512 D^{4}} .
\end{aligned}
$$

Note that as $p \rightarrow 0 \lambda_{n} \propto p^{-n}$, and as $p \rightarrow \infty \lambda_{n} \propto p^{-n-2}$.

\section{APPENDIX C. Imaginary Part of the Excitation Spectrum}

We include here a calculation of the imaginary part of $\omega_{k}$ as another example of the relative simplicity of the present method. We expand $\omega_{k}{ }^{2}$ as in (4.16) and use (4.18) to obtain

$$
\operatorname{Im} \omega_{k}=g_{2}^{1}\left(\omega_{k i}^{(0)}\right)^{-3} \operatorname{Im} \mathscr{N}^{z z(1)}\left(\mathbf{k}, \omega_{k}^{(0)}\right)+O\left(g^{2}\right) .
$$

From $(3.32 b)$, we find

$$
\begin{aligned}
\operatorname{Im} \mathscr{N}^{z z}\left(\mathbf{k}, \omega_{k}^{(0)}\right)= & \frac{1}{2} k^{4} \operatorname{Im}\left(S+M_{2}-\mu\right)+\omega_{k}^{(0)} k^{3} \operatorname{Im} \Lambda_{\mu}{ }^{z} \delta_{\mu} \\
& +\frac{1}{2} k^{5} \operatorname{Im} \Lambda_{\mu}{ }^{z} \beta_{\mu}+\frac{1}{2} k^{4} \omega_{k}^{(0)} \operatorname{Im} A \\
& +\frac{1}{4} k^{6} \operatorname{Im}(S-\mu)+k^{4} \operatorname{Im} F^{z z r},
\end{aligned}
$$

where the superscript (1) has been suppressed in (C.2). The integral over $p$ in the one-loop integrals (see (4.4)-(4.9)) in (C.2) is restricted to $p \leqslant k$ by $(4.41)$, so that 
the $k$-expansions in Appendix $B$ are not useful. Since we are interested in $\operatorname{Im} \omega_{k}$ for small $k$, we write (see (4.41))

$$
\begin{aligned}
& \delta\left(\omega_{\mathrm{k}}^{(0)}-\omega_{\mathbf{y}}^{(0)}-\omega_{\mathrm{p}}^{(0)}\right) \\
& \quad=\delta(y+p-k)+\frac{1}{8}\left(y^{3}+p^{3}-k^{3}\right) \delta^{\prime}(y+p-k)+\cdots,
\end{aligned}
$$

where $\mathbf{y}=\mathbf{p}+\mathbf{k}$. The $\delta$-function singularity in (C.3) occurs at the limit of the range of integration in the one-loop integrals of (C.2) so that these integrals are not well defined. In order to interpret these integrals, we must consider the deviation of $\omega_{k}^{(0)} / k$ from a constant in the limit of long-wavelengths. A careful limiting procedure [16] shows that, if $\omega_{k}^{(0)} / k$ is a decreasing function of $k$ for small $k$, then the one-loop integrals in (C.2) vanish and $\operatorname{Im~} \omega_{k}$ is zero. On the other hand, if $\omega_{k}^{(0)} / k$ is an increasing function of $k$ for small $k$, then the one-loop integrals in (C.2) are nonzero.

In our model calculation, $\omega_{k}^{(0)} / k$ is an increasing function of $k$ (see (3.10)), and we use the expansions $\lambda_{p}=\frac{1}{2} p-p^{3} / 16$ and (C.3) in the one-loop integrals (4.4)(4.9) to obtain by a straightforward calculation the following results for the imaginary parts evaluated at $\omega=\omega_{k}^{(0)}$

$$
\begin{aligned}
\frac{1}{2} \operatorname{Im}\left(S^{(1)}+M_{2}^{(1)}-\mu^{(1)}\right) & =-\frac{1}{960 \pi} k^{4}\left[1+\frac{11}{56} k^{2}+O\left(k^{4}\right)\right], \\
\operatorname{Im} \Lambda_{\mu}^{z(1)} \delta_{\mu} & =\frac{1}{960 \pi} k^{4}\left[1+O\left(k^{4}\right)\right], \\
\operatorname{Im} \Lambda_{\mu}^{z(1)} \beta_{\mu} & =\frac{1}{96 \pi} k^{3}\left[1-\frac{7}{40} k^{2}+O\left(k^{4}\right)\right], \\
\operatorname{Im} A^{(1)} & =-\frac{1}{96 \pi} k^{3}\left[1+\frac{1}{10} k^{2}+O\left(k^{4}\right)\right], \\
\operatorname{Im}\left(S^{(1)}-\mu^{(1)}\right) & =-\frac{1}{32 \pi} k^{2}\left[1+\frac{1}{120} k^{2}+O\left(k^{4}\right)\right], \\
\operatorname{Im} F^{z z r(1)} & =-\frac{1}{640 \pi} k^{4}\left[1-\frac{13}{56} k^{2}+O\left(k^{4}\right)\right] .
\end{aligned}
$$

If we substitute (C.4) into (C.1-C.2), we obtain

$$
\operatorname{Im} \omega_{k}=-\frac{3}{640 \pi} g k^{5}\left[1-\frac{4}{21} k^{2}+O\left(k^{4}\right)\right] .
$$

The coefficient of the leading $k^{5}$ term agrees with Beliaev [4] and the $k^{7}$ term is believed to be new. 
D. The First $\omega$-Moment Sum Rule For $\mathscr{S}(\mathbf{k}, \omega)$ AND $\mathscr{A}(\mathbf{k}, \omega)$

The sum rules involving the first $\omega$-moment of functions, $\mathscr{S}(\mathbf{k}, \omega)$ and $\mathscr{A}(\mathbf{k}, \omega)$, can be obtained from the asymptotic behavior of the respective response functions, $\mathscr{F}(\mathbf{k}, \omega)$ and $\mathscr{G}(\mathbf{k}, \omega)$. For example, the density response function $\mathscr{F}(\mathbf{k}, \omega)$ satisfies the dispersion relation

$$
\mathscr{\mathcal { F }}(\mathbf{k}, \omega)=\int_{-\infty}^{\infty} d \omega^{\prime}\left(\omega-\omega^{\prime}\right)^{-1}\left[-\pi^{-1} \operatorname{Im} \tilde{F}\left(\mathbf{k}, \omega^{\prime}\right)\right] .
$$

Since the spectral function $\operatorname{Im} \mathscr{F}(\mathbf{k}, \omega)$ is odd in $\omega$ (see (4.27)), (D.1) becomes in the asymptotic region as $\omega \rightarrow \infty$

$$
\mathscr{F}(\mathbf{k}, \omega)=2 \omega^{-2} \int_{0}^{\infty} d \omega^{\prime} \omega^{\prime} \mathscr{P}\left(\mathbf{k}, \omega^{\prime}\right)+\cdots .
$$

Comparing (D.2) with the asymptotic behavior of $\mathscr{F}(\mathbf{k}, \omega)$ as determined from the generalized Ward identity (2.31), we obtain the well known $f$ sum rule

$$
\int_{0}^{\infty} d \omega \omega \mathscr{P}(\mathbf{k}, \omega)-n k^{2} / 2 m
$$

which is quoted in (4.33).

If we separate $\mathscr{F}$ into its singular and regular parts, $\mathscr{F}=\mathscr{F}^{s}+\mathscr{F}^{r}$ with

$$
\begin{aligned}
\mathscr{F}^{s} & =\frac{1}{\epsilon} \Lambda_{\mu} G_{\mu \nu} \Lambda_{\nu}, \\
\mathscr{F}^{r} & =\frac{1}{\epsilon} F^{r},
\end{aligned}
$$

then both (D.1) and (D.2) remain valid if we replace $\mathscr{F}$ by $\mathscr{F}^{s}$ or $\mathscr{F}^{r}$ and $\mathscr{S}$ by $\mathscr{S}^{s}$ or $\mathscr{S}^{r}$, where $\mathscr{S}^{s, r}(\mathbf{k}, \omega)=-\pi^{-1} \operatorname{Im} \mathscr{F}^{s, r}(\mathbf{k}, \omega)$. We now derive a sum rule for the first $\omega$-moment of $\mathscr{P}^{s}(\mathbf{k}, \omega)$. Since $\epsilon(\mathbf{k}, \omega) \rightarrow 1$ and $\Lambda_{\mu}(\mathbf{k}, \omega) \rightarrow n_{0}^{1 / 2} \delta_{\mu}$ as $\omega \rightarrow \infty$, we see from (D.4) that the asymptotic behavior of $\mathscr{F}^{s}$ is determined by that of $n_{0} \delta_{\mu} G_{\mu \nu} \delta_{\nu}$. From the irreducible version of (2.37)-(2.39), we can obtain the following asymptotic form

$$
n_{0} \delta_{\mu} G_{\mu \nu} \delta_{\nu}=2 n_{0} \omega^{-2}\left[\epsilon_{k}+S^{\mathrm{HF}}-M_{2}^{\mathrm{HF}}-\mu\right]+\cdots,
$$

where $\epsilon_{k}=k^{2} / 2 m, S^{\mathrm{HF}}-M_{2}^{\mathrm{HF}}$ is the Hartree-Fock self energy

$$
S^{\mathrm{HF}}-M_{2}^{\mathrm{HF}}=n v(k=0)+\int \frac{d^{3} p}{(2 \pi)^{3}} v(\mathbf{k}-\mathbf{p})\left[n_{p}{ }^{\prime}-m_{p}{ }^{\prime}\right] .
$$


$n_{p}{ }^{\prime}$ is the momentum distribution (4.80), and $m_{p}{ }^{\prime}$ is the off-diagonal momentum distribution defined as in $(4.80)$ but with $\mathscr{A}(\mathbf{k}, \omega)$ replaced by $\mathscr{A}_{+-}(\mathbf{k}, \omega)$. If we compare the asymptotic behavior of $\mathscr{F}^{s}$ given by (D.6) with that given by (D.2), then we find the sum rule

$$
\int_{0}^{\infty} d \omega \omega \mathscr{F}^{s}(k, \omega)=n_{0}\left[\epsilon_{k}+S^{\mathrm{HF}}-M_{2}^{\mathrm{HF}}-\mu\right]
$$

An alternative derivation of (D.8) is to make use of a generalized Ward identity for $F^{s}$ or $F^{r}$, e.g.,

$$
F^{*}=k^{2} \omega^{-2} F^{z * *}+n_{0}^{1 / 2} \omega^{-2}\left[2 \omega \Lambda_{\mu} \beta_{\mu}-n_{0}^{1 / 2} \beta_{\mu} G_{\mu \nu}^{-1} \beta_{v}\right],
$$

which follows from (2.31), (2.33), and (2.36). Comparing (D.2) with the asymptotic behavior of $\mathscr{F}^{s}$ as determined by (D.9), we can recover the sum rule (D.9). A sum rule analogous to (D.8) has been derived for the anharmonic crystal [17], but the present derivation of (D.8) for a Bose system is believed to be new.

The first $\omega$-moment sum rule for the one-particle spectral function $\mathscr{A}(\mathbf{k}, \omega)$ can be obtained from the asymptotic behavior of the amplitude response function $\mathscr{G}_{u \nu}(\mathbf{k}, \omega)$, which satisfies the matrix dispersion relation

$$
\mathscr{G}_{\mu \nu}(\mathbf{k}, \omega)=\int_{-\infty}^{\infty_{\nu}} d \omega^{\prime}\left(\omega-\omega^{\prime}\right)^{-1} \mathscr{A}_{\mu \nu}\left(\mathbf{k}, \omega^{\prime}\right) .
$$

Since $\mathscr{A}_{\mu, v}(\mathbf{k}, \omega)$ satisfies the sum rule

$$
\int_{-\varkappa}^{x} d \omega \mathscr{A}_{\mu \nu}(\mathbf{k}, \omega)= \begin{cases} \pm 1, & \mu=\nu= \pm \\ 0, & \mu \neq \nu\end{cases}
$$

the trace of the matrix $\mathscr{G}_{\mu \nu}$ has the asymptotic form (sum over $\mu$ )

$$
\mathscr{G}_{\mu \mu}(\mathbf{k}, \omega)=2 \omega^{-2} \int_{-\infty}^{\infty} d \omega^{\prime} \omega^{\prime} \mathscr{A}\left(\mathbf{k}, \omega^{\prime}\right)+\cdots
$$

where $\mathscr{A}(\mathbf{k}, \omega)$ is given by (4.62). From (2.37)-(2.39), we can express the asymptotic behavior of $\mathscr{G}_{\mu \mu}(\mathbf{k}, \omega)$ in the form

$$
\mathscr{G}_{\mu \mu}(\mathbf{k}, \omega)=2 \omega^{-2}\left[\epsilon_{h}+S^{\mathrm{HF}}-\mu\right]+\cdots,
$$

where $S^{\mathrm{HF}}$ is the diagonal Hartree-Fock self energy,

$$
S^{\mathrm{HF}}=n v(k-0)+n_{0} v(k)+\int \frac{d^{3} p}{(2 \pi)^{3}} v(\mathbf{k}-\mathbf{p}) n_{p}{ }^{\prime}
$$


Comparing (D.12) with (D.13), we obtain the sum rule [18]

$$
\int_{-\infty}^{\infty} d \omega \omega \cdot \mathscr{L}(\mathbf{k}, \omega)=\epsilon_{k}+S^{\mathrm{HF}}-\mu
$$

The $f$ sum rule (D.3) has been explicitly verified to $O(g)$ for all $k$ in (4.57). The other two sum rules, (D.8) and (D.15), are of course satisfied in the zeroth order. However, to first order in $g$, both sides of the sum rules, (D.8) and (D.15), display large- $\omega$ or large- $p$ divergences in our model calculation. The source of these divergences can be understood by an explicit calculation of $\mathscr{S}^{r(1)}(\mathbf{k}, \omega)$. We first write

$\mathscr{F}^{r(1)}(\mathbf{k}, \omega)=\frac{\omega^{2}-\epsilon_{k}^{2}}{\omega^{2}\left(\omega^{2}-\omega_{k}^{2(s)}\right)}\left[2\left(S-M_{2}-\mu\right)+k n^{\prime}-2 k \Lambda_{\mu} \beta_{\mu}+k^{2} F^{z z r}\right]$,

where the superscript (1) has been suppressed on the right side of (D.16). Taking the imaginary part of (D.16), we note that the leading large- $\omega$ dependence arises from the $\left(S-M_{2}-\mu\right)$ term and is given by $\mathscr{S}^{r(1)}(\mathbf{k}, \omega)=\frac{1}{2} \pi^{-2} \omega^{-3 / 2}+O\left(\omega^{-5 / 2}\right)$. A similar calculation of $\mathscr{S}^{(1)}(\mathbf{k}, \omega)$ shows that the $\left(S-M_{2}-\mu\right)$ term once again dominates the large $\omega$ behavior and gives

$$
\mathscr{S}^{s(1)}(\mathbf{k}, \omega)=-\frac{1}{2} \pi^{-2} \omega^{-3 / 2}+O\left(\omega^{-5 / 2}\right) .
$$

From (D.17), we see that the first moment of $\mathscr{S}^{s(1)}(\mathbf{k}, \omega)$ is divergent. Similarly from (4.73), we see that the first moment of $\mathscr{A}^{(1)}(\mathbf{k}, \omega)$ is divergent. Following the discussion of $\mathscr{S}_{3}(k)$ in Section $4 \mathrm{C}$, we assume that (D.17) and (4.73) hold only for $\omega<1 / \tau$, where $\tau$ is the time of collision.

\section{ACKNOWLEDGMENTS}

It is a pleasure to thank Shang-keng Ma and Wayne Saslow for many useful discussions and for constant encouragement.

\section{REFERENCES}

1. J. Gavoret and P. Nozieres, Ann. Phys. (NY) 28 (1964), 349.

2. D. Pines and C.-W. Woo, Phys. Rev. Lett. 24 (1970), 1044; A. Molinari and T. Regge, Phys. Rev. Lett. 26 (1971), 1531.

3. R. A. Cowley And A. D. B. Woods, Canad. J. Phys. 49 (1971), 177; A. D. B. Woods And R. A. Cowley, Rep. Prog. Phys. 36 (1973), 1135.

4. S. T. Beliaev, Soviet Physics JETP 7 (1958), 289, 299. 
5. N. M. Hugenholtz and D. Pines, Phys. Rev. 116 (1959), 489.

6. P. C. Hohenberg and P. C. Martin, Ann. Phys. (NY) 34 (1965), 291 and references therein.

7. S. Ma and C.-W. Woo, Phys. Rev. 159 (1967), 165. See also I. Kondor and P. Szepfalusy, Acta Phys. Acad. Sci. Hungar. 24 (1968), 81.

8. S. Ma, H. Gould, and V. K. Wong, Phys. Rev. A 3 (1971), 1453.

9. H. Gould And V. K. Wong, Phys. Rev. Lett. 27 (1971), 301.

10. V. K. WONG AND H. Gould, Phys. Lett. 39A (1972), 331.

11. S. MA, J. Math. Phys. 12 (1971), 2157; S. MA, Phys. Rev. A5 (1972), 2632.

12. F. De Pasquale, G. Jona-Lasino, and E. Tabet, Ann. Phys. (NY) 33 (1965), 381.

13. See for example A. A. Abrikosov et al., "Methods of Quantum Field Theory in Statistical Physics," Prentice Hall, Englewood Cliffs, NJ, 1963.

14. A. Miller, D. Pines, And P. Nozieres, Phys. Rev. 127 (1962), 1452.

15. P. Kieban, Phys. Rev. Lett. 27 (1971), 657.

16. M. Nelkin, Phys. Rev. 127 (1962), 979.

17. V. Ambegaoker, J. M. Conway, and G. Baym, in "Lattice Dynamics" (R. F. Wallis, Ed.), p. 261, Pergamon, London, 1965.

18. H. WaGner, Z. Physik 195 (1966), 273. 